\title{
Design of inerter-based multi-actuator systems for vibration control of adjacent structures
}

\author{
F. Palacios-Quiñonero ${ }^{\text {a }, ~ J . ~ R u b i o ́-M a s s e g u ́ a ~}{ }^{\text {, J. M. Rossell }}{ }^{\mathrm{a}}$, H. R. Karimi ${ }^{\mathrm{b}, *}$ \\ ${ }^{a}$ Dept. of Mathematics, Universitat Politècnica de Catalunya (UPC), Escola Politècnica Superior d'Enginyeria de \\ Manresa (EPSEM), Av. Bases de Manresa, 61-73, 08242 Manresa, Spain \\ ${ }^{b}$ Dept. of Mechanical Engineering, Politecnico di Milano, via La Masa 1, 20156 Milan, Italy
}

\begin{abstract}
Distributed multi-actuator systems can provide effective solutions for mitigating the vibrational response of large structures. In this paper, we present a computational strategy to design inerter-based multi-actuation systems for the seismic protection of adjacent structures. The proposed approach allows considering both interstory and interbuilding Tuned Mass-Inerter Damper (TMID) actuators, and aims at simultaneously reducing the vibrational response of the individual buildings and avoiding the interbuilding impacts. The tuning procedure is based on an $H_{\infty}$ costfunction and uses a constrained global-optimization solver to compute parameter configurations with high-performance characteristics. To illustrate the main features of this work, two different Tuned Inerter Damper (TID) multi-actuator schemes are considered for the seismic protection of a particular multi-story two-building system. A multi-actuator Tuned Mass Damper (TMD) system is also designed and is taken as a reference in the performance assessment. The obtained results demonstrate the flexibility and effectiveness of the proposed design methodology, and clearly show the superior performance and robustness of the TID actuation systems.
\end{abstract}

Keywords: tuned inerter dampers, structural vibration control, multi-structure systems, multi-actuation systems, seismic control

\section{Introduction}

Inerters are a type of mechanical elements that allow modifying the inertial characteristics of mechanical systems without introducing significant changes in the system mass. The ideal linear inerter is a massless two-terminal element that produces a resistant force of the form $F(t)=b \ddot{r}(t)$, where $\ddot{r}(t)$ is the relative acceleration through the inerter terminals and $b \in \mathbb{R}$ is a constant, which is called inertance and has dimension of mass [1]. In practical implementations, the ratio from the inertance to the inerter physical mass can attain large values, producing an apparent massamplification effect that can exceed two orders of magnitude [2]. Physical realizations of inerters

\footnotetext{
* Corresponding author

Email addresses: francisco.palacios@upc.edu (F. Palacios-Quiñonero), josep.rubio@upc.edu (J. Rubió-Massegú), josep.maria.rossell@upc.edu (J. M. Rossell), hamidreza. karimi@polimi . it (H. R. Karimi)
}

Preprint submitted to Journal of the Franklin Institute 
with an approximate linear behavior have been carried out by means of flywheels with rack-andpinion and ball-screw mechanisms [2,3]. Other implementations include fluid inerters [4-6] and more advanced designs, as planetary flywheel inerters [7] and inerters with variable inertance [8-10]. Since their introduction in the earlies 2000s, inerters have been attracting an increasing research interest. Instances of innovative applications can be found in a wide variety of technical and scientific fields, such as automotive suspensions [11-14], vibrational isolation systems [10, 15-19], airplane landing systems [20], biped walking robots [21], biomedical muscular models [22] and design of metamaterials [23]. In structural vibration control, the usage of inerters provides the possibility of producing large inertial forces by means of light and compact actuation devices. Moreover, the two-terminal character of inerter elements allows considering new and more sophisticated passive actuation layouts [24, 25]. Recent research works in this field include novel inerter-based strategies to mitigate the vibrational response of buildings [26-32], bridges [33, 34], liquid storage tanks [35] and wind turbines [36, 37]. Also, an increasing attention is being paid to combined strategies for vibration control and energy harvesting [38-41].

Seismic protection of closely adjacent buildings requires paying attention to both reducing the vibrational response of the individual buildings and avoiding interbuilding collisions (pounding). Advanced solutions to this complex problem can be obtained by means of distributed multiactuator schemes that combine interstory and interbuilding actuation devices [42, 43]. In this context, inerters constitute a very attractive option due to their two-terminal character, light weight and reduced size. Furthermore, the positive behavior of inerter-based multi-actuator systems has been reported in single-building studies [44].

Motivated by these facts, the present paper is focused on exploring the potential benefits of using Tuned Inerter Dampers (TIDs) in distributed multi-actuator schemes for vibration control of multi-building systems. In order to provide a detailed presentation of the modeling aspects, the discussion has been constrained to the two-building case and the Tuned Mass-Inerter Damper (TMID) actuator layout. Focusing on two-building systems facilitates a clear presentation of the building interactions and the combined effect of interstory and interbuilding actuation devices (see Fig. 1). The selected TMID actuator layout includes a series arrangement of a mass-springdamper and an inerter element (see Fig. 2). This particular kind of passive configuration is a natural extension of classical Tuned Mass Dampers (TMDs), and can be used to define both TID and TMD vibration control strategies [26-32].

It is worth highlighting that, due to the complexity and high dimensionality of the considered problem, modeling and computational aspects are elements of singular relevance to obtain effective solutions. In this line, the paper makes a twofold contribution: (i) to provide an explicit parametric model for the dynamical response of two-building systems equipped with multiple interstory and/or interbuilding TMID actuation devices, and (ii) to design a computationally effective tuning procedure that allows obtaining suitable parameter values for a given multi-device TMID actuation scheme. The proposed tuning procedure is based on a constrained optimization problem with an $H_{\infty}$ cost-function, which can be numerically implemented by using off-the-shelf optimization solvers. Specifically, a Particle Swarm algorithm [45-47] with bound constraints is used in this work with positive results. The effectiveness of the proposed approach is demonstrated by means of a particular multi-story two-building structure equipped with two different TID multiactuator schemes. A TMD multi-actuator system is also considered, and it is taken as a natural 
reference in the performance assessment.

The rest of the paper is organized as follows: In Section 2, a general matrix model for a twobuilding system equipped with multiple interstory and/or interbuilding TMID devices is provided. In Section 3, the tuning procedure is presented and applied to obtain suitable parameter sets for the considered actuation schemes. Also, the corresponding frequency responses are computed and compared. In Section 4, the time-response characteristics corresponding to a medium-size and a strong near-fault seismic excitation are discussed. Finally, in Section 5, some conclusions and future research directions are briefly presented.

\section{Mathematical model}

\subsection{Uncontrolled two-building system}

Let us consider a multi-story two-building system as the one schematically displayed in Fig. 1a. The lateral motion of the building $\mathscr{B}^{(j)}$ can be described by the second-order model

$$
\mathbf{M}^{(j)} \ddot{\mathbf{q}}^{(j)}(t)+\mathbf{C}^{(j)} \dot{\mathbf{q}}^{(j)}(t)+\mathbf{K}^{(j)} \mathbf{q}^{(j)}(t)=-\mathbf{M}^{(j)}[\mathbf{1}]_{n_{j} \times 1} \ddot{w}_{g}(t),
$$

where $n_{j}$ is the number of stories; $\mathbf{q}^{(j)}(t)=\left[q_{1}^{j}(t), \ldots, q_{n_{j}}^{j}(t)\right]^{T} \in \mathbb{R}^{n_{j} \times 1}$ is the vector of story displacements with respect to the ground; $\mathbf{M}^{(j)} \in \mathbb{R}^{n_{j} \times n_{j}}, \mathbf{C}^{(j)} \in \mathbb{R}^{n_{j} \times n_{j}}$ and $\mathbf{K}^{(j)} \in \mathbb{R}^{n_{j} \times n_{j}}$ are the building mass matrix, damping matrix and stiffness matrix, respectively; $[\mathbf{1}]_{n_{j} \times 1}$ is a column vector of dimension $n_{j}$ with all its entries equal to 1 ; and $\ddot{w}_{g}(t)$ is the ground acceleration disturbance. The mass and stiffness building matrices of building $\mathscr{B}^{(j)}$ have the following form:

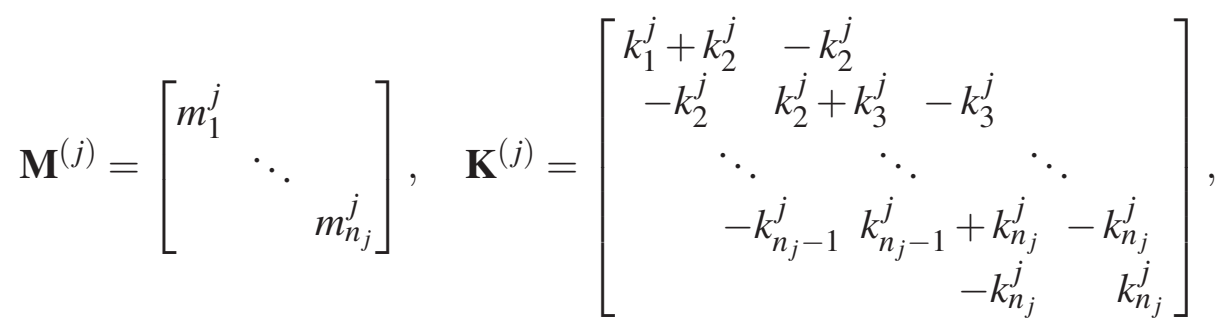

where $m_{i}^{j}$ and $k_{i}^{j}$ are the respective mass and stiffness coefficients of the $i$-th story in $\mathscr{B}^{(j)}$. When the values of the story damping-coefficients $c_{i}^{j}$ are known, a tridiagonal damping-matrix $\mathbf{C}^{(j)}$ can be obtained by replacing the coefficients $k_{i}^{j}$ in $\mathbf{K}^{(j)}$ by the corresponding damping coefficients $c_{i}^{j}$, $i=1, \ldots, n_{j}$. Alternatively, an approximate damping matrix $\mathbf{C}^{(j)}$ can be computed when the values of the story damping-coefficients $c_{i}^{j}$ are not available [48].

The overall motion of the non-actuated two-building system, can be described by the secondorder model

$$
\mathbf{M} \ddot{\mathbf{q}}(t)+\mathbf{C} \dot{\mathbf{q}}(t)+\mathbf{K q}(t)=-\mathbf{M}[\mathbf{1}]_{n \times 1} \ddot{w}_{g}(t),
$$

where $n=n_{1}+n_{2}$ is the total number of stories; $\mathbf{q}(t) \in \mathbb{R}^{n \times 1}$ is the overall vector of story displacements with respect to the ground, which can be written in the form

$$
\mathbf{q}(t)=\left[\begin{array}{l}
\mathbf{q}^{(1)}(t) \\
\mathbf{q}^{(2)}(t)
\end{array}\right] ;
$$




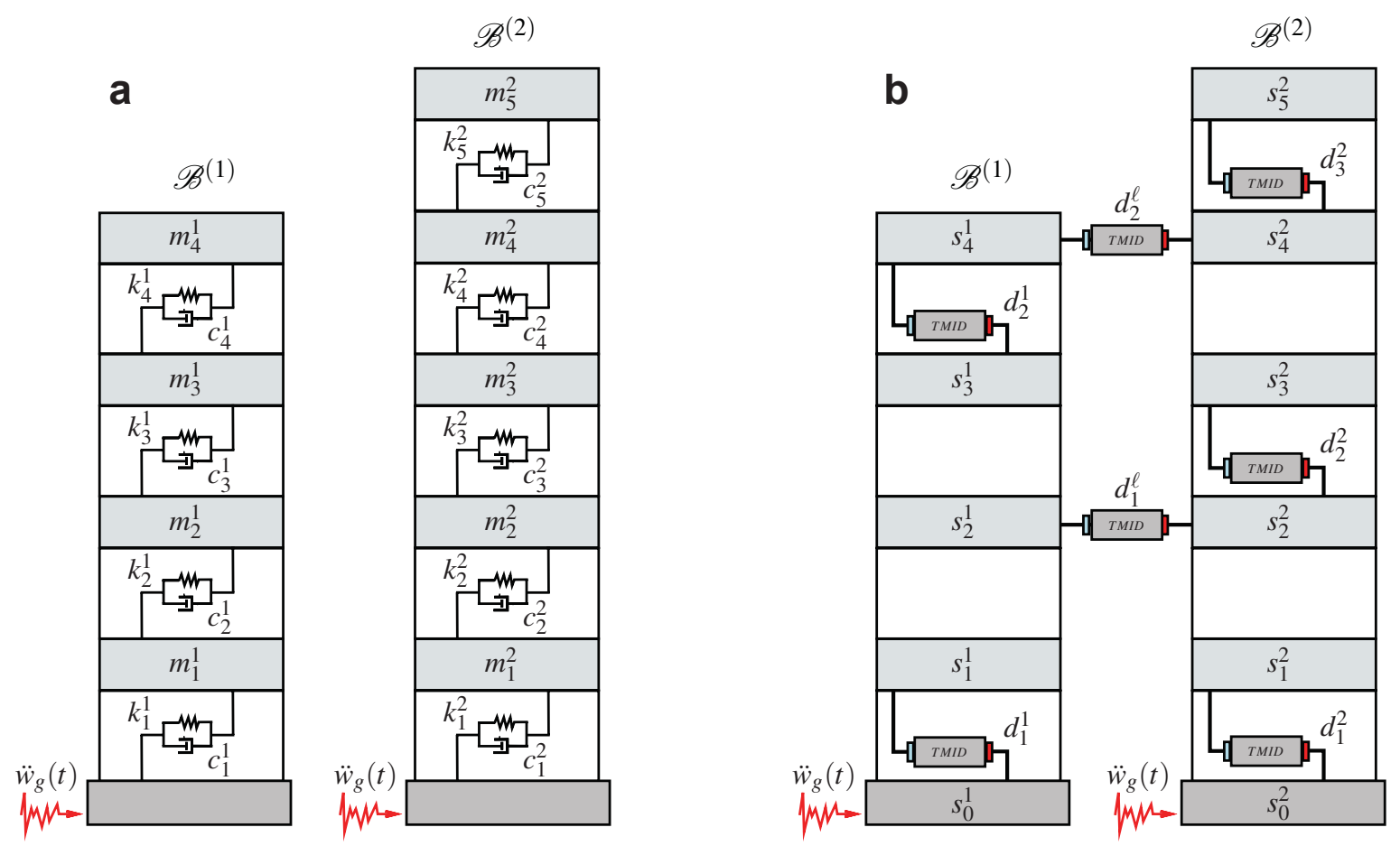

Figure 1: Multi-story two-building system: (a) Mechanical elements of the non-actuated system. (2) Distributed multi-actuator scheme with interstory and interbuilding actuation devices.

and the overall mass, damping, and stiffness matrices have, respectively, the following blockdiagonal structure:

$$
\mathbf{M}=\left[\begin{array}{cc}
\mathbf{M}^{(1)} & {[\mathbf{0}]_{n_{1} \times n_{2}}} \\
{[\mathbf{0}]_{n_{2} \times n_{1}}} & \mathbf{M}^{(2)}
\end{array}\right], \mathbf{C}=\left[\begin{array}{cc}
\mathbf{C}^{(1)} & {[\mathbf{0}]_{n_{1} \times n_{2}}} \\
{[\mathbf{0}]_{n_{2} \times n_{1}}} & \mathbf{C}^{(2)}
\end{array}\right], \mathbf{K}=\left[\begin{array}{cc}
\mathbf{K}^{(1)} & {[\mathbf{0}]_{n_{1} \times n_{2}}} \\
{[\mathbf{0}]_{n_{2} \times n_{1}}} & \mathbf{K}^{(2)}
\end{array}\right],
$$

where $[\mathbf{0}]_{m \times n}$ is a null matrix of dimension $m \times n$.

\subsection{TMID model}

Let us consider the TMID schematically depicted in Fig. 2, which includes a series arrangement of a mass-spring-damper system and an inerter element. The dynamical behavior of this passive actuation device can be described by the following second-order model:

$$
(\hat{m}+b) \ddot{y}(t)-b \ddot{q}_{r}(t)+\hat{c} \dot{y}(t)-\hat{c} \dot{q}_{c}(t)+\hat{k} y(t)-\hat{k} q_{c}(t)=-\hat{m} \ddot{w}_{g}(t),
$$

where $y(t)$ is the mass displacement and $q_{c}(t), q_{r}(t)$ are the displacements of the case and rod, respectively. All the displacements are taken with respect to the ground, which is a non-inertial reference frame subject to the ground acceleration $\ddot{w}_{g}(t)$. As indicated in the figure, the mass, damping and stiffness coefficients are denoted by $\hat{m}, \hat{c}$ and $\hat{k}$, respectively, and $b$ is the inertance coefficient. It should be noted that the proposed TMID element has an asymmetric layout: the 

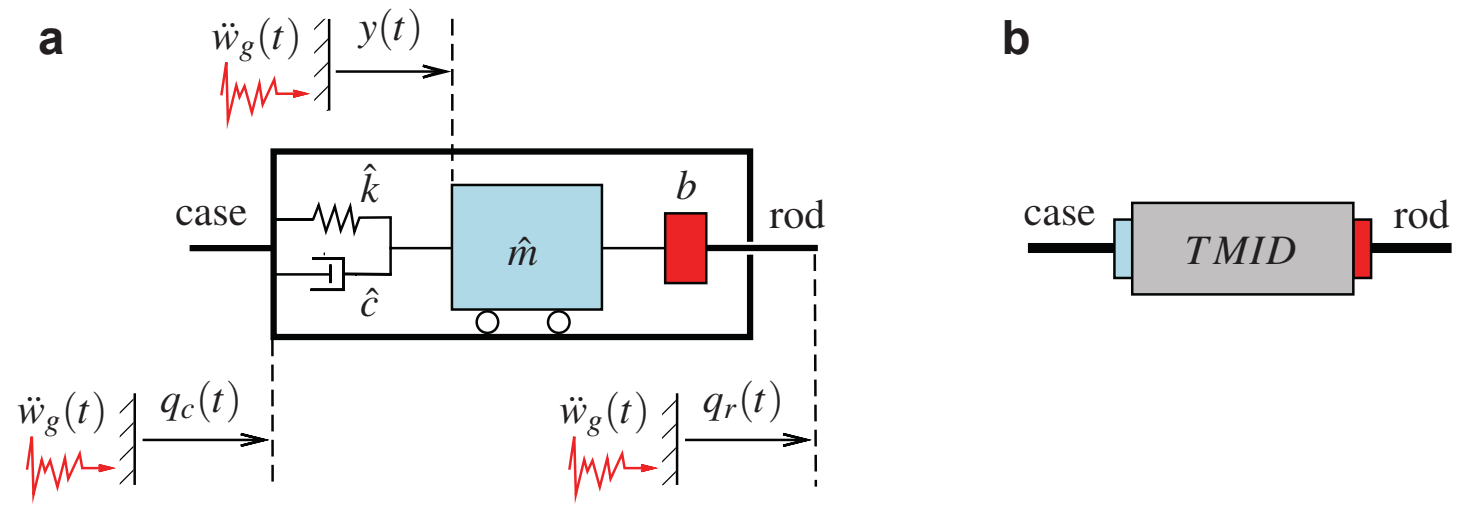

Figure 2: Tuned Mass-Inerter Damper (TMID): (a) Schematic setup. (b) Iconic representation.

spring and damper elements are connected to the case, and the inerter is linked to the rod. For the TMID iconic representation in Fig. 2b, we will assume that the left-hand terminal (marked in light blue) corresponds to the case, and the rod is associated to the right-hand terminal (marked in red). In the present work, two different kinds of TMID implementations are considered: (i) interstory TMIDs, which are allocated between consecutive stories of the same building (see Fig. 3); and (ii) interbuilding TMIDs, which act as interbuilding linking elements and are implemented between stories located at the same level in the adjacent buildings (see Fig. 5). For interstory TMIDs, we will assume that the case terminal is connected to the upper story and the rod terminal is connected to the lower story. Hence, for an interstory TMID implemented at the $i$-th level in building $\mathscr{B}^{(j)}$ between the stories $s_{i}^{j}$ and $s_{i-1}^{j}$, we will have $q_{c}(t)=q_{i}^{j}(t)$ and $q_{r}(t)=q_{i-1}^{j}(t)$. For interbuilding TMIDs, we set the convention that the case terminal is connected to the left-hand building and the rod terminal is linked to the right-hand building. Thus, for an interbuilding TMID implemented at the $i$-th story-level between the stories $s_{i}^{1}$ and $s_{i}^{2}$, we will have $q_{c}(t)=q_{i}^{1}(t)$ and $q_{r}(t)=q_{i}^{2}(t)$.

Remark 1. The proposed TMID element allows conducting a unified treatment of both Tuned Mass Damper (TMD) and Tuned Inerter Damper (TID) elements. Specifically, a TMD element with mass $\hat{m}>0$ is obtained by setting $b=0$. Analogously, a TID element with inertance $b>0$ can be obtained by setting $\hat{m}=0$. When convenient, the iconic representation in Figure $2 b$ with the label TID will be used in graphical representations of TID multi-actuation systems (see Fig. 6).

\subsection{Systems of interstory TMIDs and extended dynamical model of actuated buildings}

Let us assume that the building $\mathscr{B}^{(j)}$ is equipped with a system $D^{(j)}=\left[d_{1}^{j}, \ldots, d_{\hat{n}_{j}}^{j}\right]$ of $\hat{n}_{j} \geq 0$ interstory TMIDs implemented at selected interstory levels. In the trivial case $\hat{n}_{j}=0$, there are no interstory actuation devices implemented in $\mathscr{B}^{(j)}$, and we say that this building has a nonactuated configuration. For an actuated building $\mathscr{B}^{(j)}$ equipped with a system of $\hat{n}_{j}>0$ interstory TMIDs, we will suppose that each interstory level can contain at most one interstory actuation device, and will indicate the position of the interstory device $d_{i}^{j}$ by means of the associated upper story-level position $p_{i}^{j}$. Thus, for example, the actuation scheme presented in Fig. $1 \mathrm{~b}$ contains two 

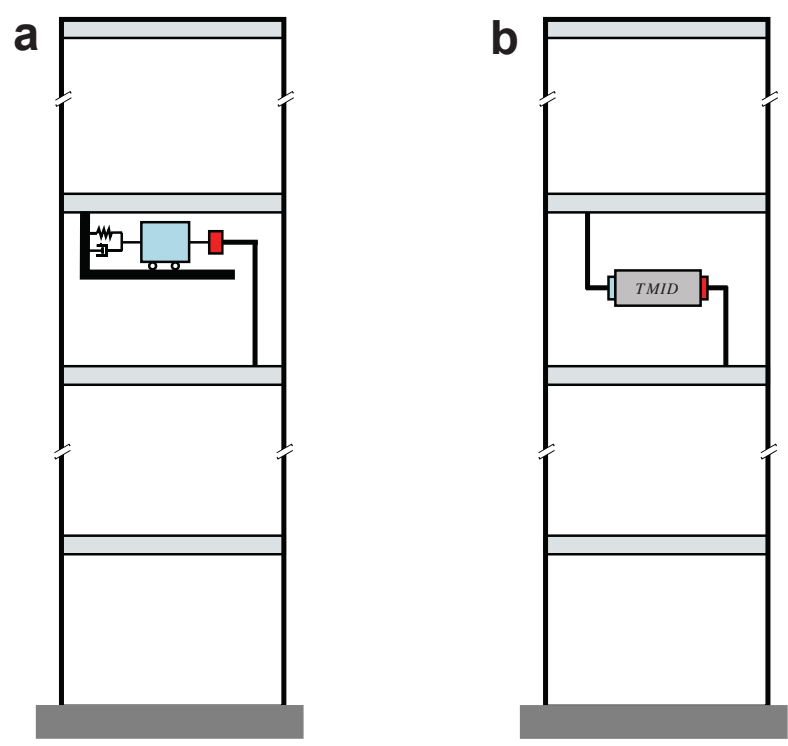

Figure 3: Interstory TMID implemented between consecutive stories of the same building: (a) Schematic setup. (b) Iconic representation.

systems of interstory TMIDs: $D^{(1)}=\left[d_{1}^{1}, d_{2}^{1}\right]$ and $D^{(2)}=\left[d_{1}^{2}, d_{2}^{2}, d_{3}^{2}\right]$. The actuation device $d_{2}^{1}$ is the second interstory TMID of building $\mathscr{B}^{(1)}$ and is placed at the building level $p_{2}^{1}=4 ; d_{3}^{2}$ is the third interstory TMID of building $\mathscr{B}^{(2)}$ and is located at the building level $p_{3}^{2}=5$. The parameters corresponding to the interstory TMID $d_{i}^{j}$ are denoted by $\hat{m}_{i}^{j}, \hat{c}_{i}^{j}, \hat{k}_{i}^{j}$ and $b_{i}^{j}$; and $y_{i}^{j}(t)$ represents the displacement of the TMID mass with respect to the ground (see Fig. 4). To describe the overall characteristics of an interstory TMID system $D^{(j)}$ with $\hat{n}_{j}>0$, we consider the diagonal matrices

$$
\hat{\mathbf{M}}^{(j)}=\left[\begin{array}{lll}
\hat{m}_{1}^{j} & & \\
& \ddots & \\
& & \hat{m}_{\hat{n}_{j}}^{j}
\end{array}\right], \hat{\mathbf{C}}^{(j)}=\left[\begin{array}{ccc}
\hat{c}_{1}^{j} & & \\
& \ddots & \\
& & \hat{c}_{\hat{n}_{j}}^{j}
\end{array}\right], \hat{\mathbf{K}}^{(j)}=\left[\begin{array}{ccc}
\hat{k}_{1}^{j} & & \\
& \ddots & \\
& & \hat{k}_{\hat{n}_{j}}^{j}
\end{array}\right], \mathbf{B}^{(j)}=\left[\begin{array}{lll}
b_{1}^{j} & & \\
& \ddots & \\
& & b_{\hat{n}_{j}}^{j}
\end{array}\right]
$$

and the vector of mass displacements $\mathbf{y}^{(j)}(t)=\left[y_{1}^{j}(t), \ldots, y_{\hat{n}_{j}}^{j}(t)\right]^{T}$. The location of the interstory TMIDs included in $D^{(j)}$ are specified by the list of actuator positions $P^{(j)}=\left[p_{1}^{j}, \ldots, p_{\hat{n}_{j}}^{j}\right]$. In order to model the dynamical effect of the interstory actuator positions, we introduce two incidence matrices $\mathbf{P}_{c}^{(j)} \in \mathbb{R}^{n_{j} \times \hat{n}_{j}}$ and $\mathbf{P}_{r}^{(j)} \in \mathbb{R}^{n_{j} \times \hat{n}_{j}}$, which indicate the story-levels where the case and rod terminals are respectively attached. The case-placement matrix $\mathbf{P}_{c}^{(j)}$ contains the columns of the identity matrix $\mathbf{I}_{n_{j}}$ indicated in the list of positions $P^{(j)}$. The rod-placement matrix $\mathbf{P}_{r}^{(j)}$ can be obtained by considering the submatrix of $\mathbf{I}_{n_{j}+1}$ formed by the rows in positions $2, \ldots, n_{j}+1$ and the columns indicated in $P^{(j)}$. Thus, for instance, the lists of actuator positions corresponding to the interstory TMID systems $D^{(1)}$ and $D^{(2)}$ of the actuation system displayed in Fig. $1 \mathrm{~b}$ are $P^{(1)}=[1,4]$ and $P^{(2)}=[1,3,5]$, respectively. The associated case- and rod-placement matrices 


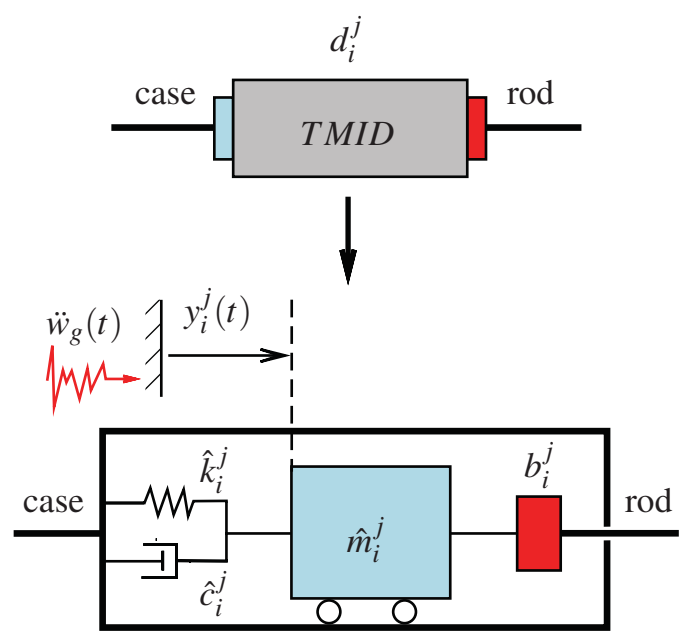

Figure 4: Notations for the interstory TMID $d_{i}^{j}$. For the interbuilding TMID $d_{i}^{\ell}$, the superscript $j$ is substituted by the letter $\ell$. In this case, the TMID mass, damping, stiffness and inertance coefficients are denoted by $\hat{m}_{i}^{\ell}, \hat{c}_{i}^{\ell}, \hat{k}_{i}^{\ell}$ and $b_{i}^{\ell}$, respectively, and the mass displacement is represented by $y_{i}^{\ell}(t)$.

have the following form:

$$
\mathbf{P}_{c}^{(1)}=\left[\begin{array}{ll}
1 & 0 \\
0 & 0 \\
0 & 0 \\
0 & 1
\end{array}\right], \mathbf{P}_{r}^{(1)}=\left[\begin{array}{cc}
0 & 0 \\
0 & 0 \\
0 & 1 \\
0 & 0
\end{array}\right] ; \quad \mathbf{P}_{c}^{(2)}=\left[\begin{array}{lll}
1 & 0 & 0 \\
0 & 0 & 0 \\
0 & 1 & 0 \\
0 & 0 & 0 \\
0 & 0 & 1
\end{array}\right], \mathbf{P}_{r}^{(2)}=\left[\begin{array}{lll}
0 & 0 & 0 \\
0 & 1 & 0 \\
0 & 0 & 0 \\
0 & 0 & 1 \\
0 & 0 & 0
\end{array}\right] .
$$

The dynamical behavior of an actuated building $\mathscr{B}^{(j)}$ equipped with a system $D^{(j)}$ of $\hat{n}_{j}>0$ interstory TMIDs can be described by the second-order model

$$
\tilde{\mathbf{M}}^{(j)} \ddot{\tilde{\mathbf{q}}}^{(j)}(t)+\tilde{\mathbf{C}}^{(j)} \dot{\tilde{\mathbf{q}}}(t)+\tilde{\mathbf{K}}^{(j)} \tilde{\mathbf{q}}^{(j)}(t)=-\tilde{\mathbf{M}}_{w}^{(j)}[\mathbf{1}]_{\tilde{n}_{j} \times 1} \ddot{w}_{g}(t),
$$

where $\tilde{\mathbf{q}}^{(j)}(t) \in \mathbb{R}^{\tilde{n}_{j} \times 1}$ is an extended displacement vector with the following form:

$$
\tilde{\mathbf{q}}^{(j)}(t)=\left[\begin{array}{c}
\mathbf{q}^{(j)}(t) \\
\mathbf{y}^{(j)}(t)
\end{array}\right]=\left[q_{1}^{j}(t), \ldots, q_{n_{j}}^{j}(t), y_{1}^{j}(t), \ldots, y_{\hat{n}_{j}}^{j}(t)\right]^{T},
$$

and $\tilde{n}_{j}=n_{j}+\hat{n}_{j}$ is the number of degrees-of-freedom corresponding to the actuated building $\mathscr{B}^{(j)}$. The generalized mass-matrix $\tilde{\mathbf{M}}^{(j)} \in \mathbb{R}^{\tilde{n}_{j} \times \tilde{n}_{j}}$ has the following block structure:

$$
\tilde{\mathbf{M}}^{(j)}=\left[\begin{array}{cc}
\mathbf{M}^{(j)}+\mathbf{P}_{r}^{(j)} \mathbf{B}^{(j)}\left\{\mathbf{P}_{r}^{(j)}\right\}^{T} & -\mathbf{P}_{r}^{(j)} \mathbf{B}^{(j)} \\
-\left\{\mathbf{P}_{r}^{(j)} \mathbf{B}^{(j)}\right\}^{T} & \hat{\mathbf{M}}^{(j)}+\mathbf{B}^{(j)}
\end{array}\right],
$$


where $\mathbf{M}^{(j)}$ is the mass matrix of $\mathscr{B}^{(j)}, \hat{\mathbf{M}}^{(j)}$ and $\mathbf{B}^{(j)}$ are the mass and inertance matrices of the interstory actuation system $D^{(j)}$, and $\mathbf{P}_{r}^{(j)}$ is the rod-placement matrix of $D^{(j)}$. The extended damping-matrix $\tilde{\mathbf{C}}^{(j)} \in \mathbb{R}^{\tilde{n}_{j} \times \tilde{n}_{j}}$ can be written in the form

$$
\tilde{\mathbf{C}}^{(j)}=\left[\begin{array}{cc}
\mathbf{C}^{(j)}+\mathbf{P}_{c}^{(j)} \hat{\mathbf{C}}^{(j)}\left\{\mathbf{P}_{c}^{(j)}\right\}^{T} & -\mathbf{P}_{c}^{(j)} \hat{\mathbf{C}}^{(j)} \\
-\left\{\mathbf{P}_{c}^{(j)} \hat{\mathbf{C}}^{(j)}\right\}^{T} & \hat{\mathbf{C}}^{(j)}
\end{array}\right],
$$

where $\mathbf{C}^{(j)}$ is the damping matrix of $\mathscr{B}^{(j)}, \hat{\mathbf{C}}^{(j)}$ is the damping matrix of the interstory actuation system $D^{(j)}$, and $\mathbf{P}_{c}^{(j)}$ is the case-placement matrix of $D^{(j)}$. Analogously, the extended stiffnessmatrix $\tilde{\mathbf{K}}^{(j)} \in \mathbb{R}^{\tilde{n}_{j} \times \tilde{n}_{j}}$ can be written as

$$
\tilde{\mathbf{K}}^{(j)}=\left[\begin{array}{cc}
\mathbf{K}^{(j)}+\mathbf{P}_{c}^{(j)} \hat{\mathbf{K}}^{(j)}\left\{\mathbf{P}_{c}^{(j)}\right\}^{T} & -\mathbf{P}_{c}^{(j)} \hat{\mathbf{K}}^{(j)} \\
-\left\{\mathbf{P}_{c}^{(j)} \hat{\mathbf{K}}^{(j)}\right\}^{T} & \hat{\mathbf{K}}^{(j)}
\end{array}\right],
$$

where $\mathbf{K}^{(j)}$ is the stiffness matrix of $\mathscr{B}^{(j)}$ and $\hat{\mathbf{K}}^{(j)}$ is the stiffness matrix of $D^{(j)}$. Finally, the extended mass-matrix $\tilde{\mathbf{M}}_{w}^{(j)} \in \mathbb{R}^{\tilde{n}_{j} \times \tilde{n}_{j}}$ has the form

$$
\tilde{\mathbf{M}}_{w}^{(j)}=\left[\begin{array}{cc}
\mathbf{M}^{(j)} & {[\mathbf{0}]_{n_{j} \times \hat{n}_{j}}} \\
{[\mathbf{0}]_{\hat{n}_{j} \times n_{j}}} & \hat{\mathbf{M}}^{(j)}
\end{array}\right] .
$$

For a non-actuated building $\mathscr{B}^{(j)}$, the number of interstory actuation devices is $\hat{n}_{j}=0$. A unified treatment of the actuated and non-actuated cases can be performed by considering degenerate empty matrices with at least one null-dimension. The basic idea consists in obtaining first a suitable block-structure for the system matrices of the actuated case with $\hat{n}_{j}>0$ and, next, deriving the system matrices corresponding to the non-actuated case by setting $\hat{n}_{j}=0$ and removing all the degenerated empty matrices. Thus, after setting $\hat{n}_{j}=0$, we observe that the column vector $\mathbf{y}^{(j)}(t)$ and the matrices $\hat{\mathbf{M}}^{(j)}, \hat{\mathbf{C}}^{(j)}, \hat{\mathbf{K}}^{(j)}, \mathbf{B}^{(j)}, \mathbf{P}_{c}^{(j)}$ and $\mathbf{P}_{r}^{(j)}$ all become degenerate empty matrices and, after removing them from the block-matrices in Eqs. (10)-(14), we obtain

$$
\tilde{\mathbf{q}}^{(j)}(t)=\mathbf{q}^{(j)}(t), \quad \tilde{\mathbf{M}}^{(j)}=\tilde{\mathbf{M}}_{w}^{(j)}=\mathbf{M}^{(j)}, \quad \tilde{\mathbf{C}}^{(j)}=\mathbf{C}^{(j)}, \quad \tilde{\mathbf{K}}^{(j)}=\mathbf{K}^{(j)},
$$

and the model of the actuated case in Eq. (9) reduces to the non-actuated model given in Eq. (1).

Remark 2. It should be noted that the introduction of the inerter elements in the actuation devices leads to considering two different kinds of mass matrices: (i) the generalized mass-matrix $\tilde{\mathbf{M}}^{(j)}$ in Eq. (11), which includes the building masses, the TMID masses and the inertance coefficients; and (ii) the extended mass-matrix $\tilde{\mathbf{M}}_{w}^{(j)}$ in Eq. (14), which only includes the building and TMID masses, and is used to introduce the inertial forces in the dynamical model.

Remark 3. It is worth highlighting that empty matrices are not just a convenient algebraic resource but also a very effective computational tool, which is implemented in standard computation programs as Matlab or Octave. 

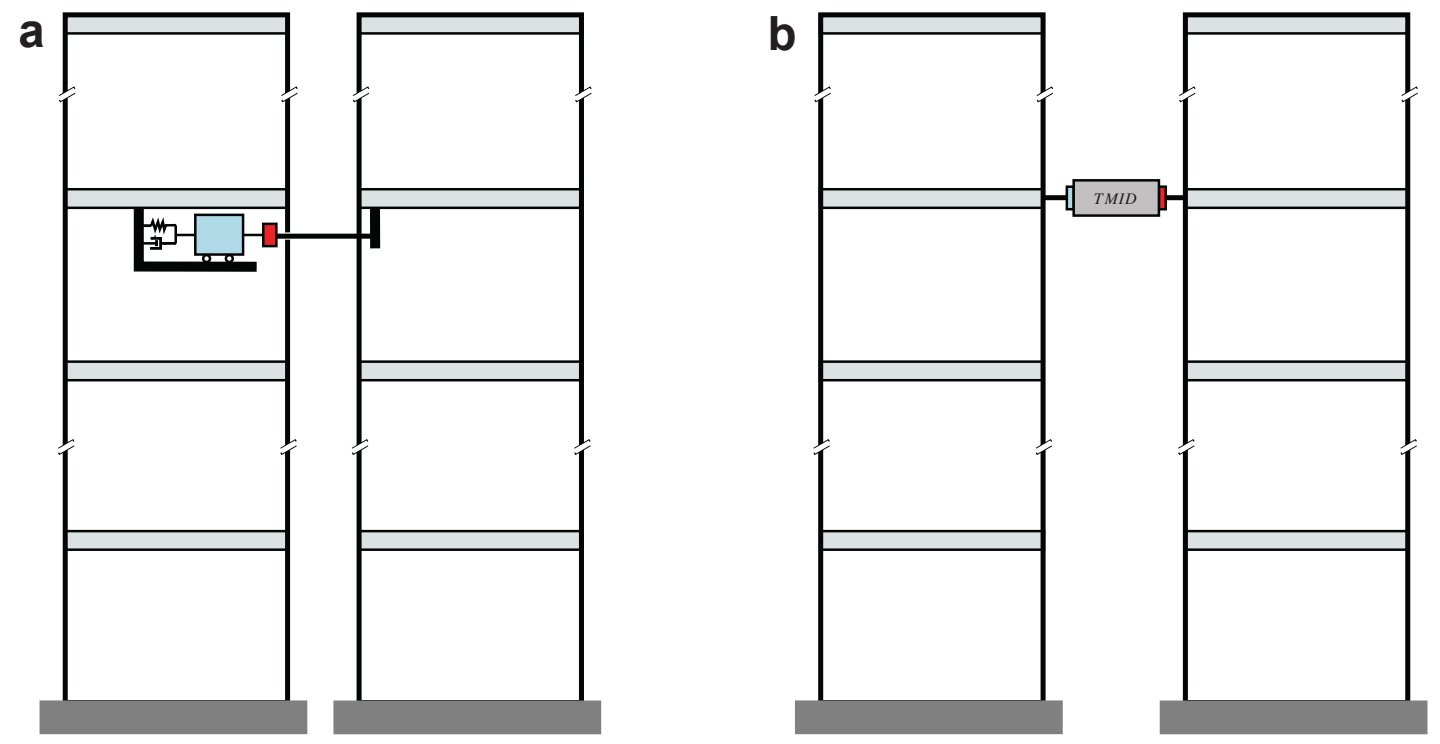

Figure 5: Interbuilding TMID implemented between stories located at the same level in the adjacent buildings: (a) Schematic setup. (b) Iconic representation.

\subsection{Systems of interbuilding TMIDs and extended dynamical model for linked actuation schemes}

Let us now consider a system $D^{(\ell)}=\left[d_{1}^{\ell}, \ldots, d_{\hat{n}_{\ell}}^{\ell}\right]$ with $\hat{n}_{\ell} \geq 0$ TMIDs implemented at selected interbuilding story-levels of the two-building system. In the trivial case $\hat{n}_{\ell}=0$, there are no actuation devices implemented between $\mathscr{B}^{(1)}$ and $\mathscr{B}^{(2)}$, and we say that the buildings have an unlinked configuration. For a linked configuration with $\hat{n}_{\ell}>0$ interbuilding TMIDs, we assume that at most one TMID can be implemented at each interbuilding story-level. In this case, we will have $0<\hat{n}_{\ell} \leq n_{\ell}$, where $n_{\ell}=\min \left(n_{1}, n_{2}\right)$ is the overall number of linkable interbuilding story-levels in the two-building system. To indicate the position of the interbuilding device $d_{i}^{\ell}$, we will use the corresponding story-level position-value $p_{i}^{\ell}$. Thus, for example, the linked actuation scheme presented in Fig. $1 \mathrm{~b}$ contains a system $D^{(\ell)}=\left[d_{1}^{\ell}, d_{2}^{\ell}\right]$ with $\hat{n}_{\ell}=2$ interbuilding TMIDs. The actuation device $d_{1}^{\ell}$ is the first interbuilding TMID and it is implemented at the story-level position $p_{1}^{\ell}=2$; the second interbuilding TMID $d_{2}^{\ell}$ is implemented at the story-level position $p_{2}^{\ell}=4$. Adopting a set of notations analogous to those used for the interstory TMIDs (see Fig. 4), we will denote the parameters corresponding to the interbuilding actuation device $d_{i}^{\ell}$ by $\hat{m}_{i}^{\ell}, \hat{c}_{i}^{\ell}, \hat{k}_{i}^{\ell}$ and $b_{i}^{\ell}$. Similarly, we will represent the displacement of the TMID mass $\hat{m}_{i}^{\ell}$ with respect to the ground by $y_{i}^{\ell}(t)$. The overall characteristics of an interbuilding actuation system $D^{(\ell)}$ with $\hat{n}_{\ell}>0$ interbuilding TMIDs can be described by the diagonal matrices

$$
\hat{\mathbf{M}}^{(\ell)}=\left[\begin{array}{ccc}
\hat{m}_{1}^{\ell} & & \\
& \ddots & \\
& & \\
& & \hat{m}_{\hat{n}_{\ell}}^{\ell}
\end{array}\right], \hat{\mathbf{C}}^{(\ell)}=\left[\begin{array}{ccc}
\hat{c}_{1}^{\ell} & & \\
& \ddots & \\
& & \hat{c}_{\hat{n}_{\ell}}^{\ell}
\end{array}\right], \hat{\mathbf{K}}^{(\ell)}=\left[\begin{array}{ccc}
\hat{k}_{1}^{\ell} & & \\
& \ddots & \\
& & \hat{k}_{\hat{n}_{\ell}}^{\ell}
\end{array}\right], \mathbf{B}^{(\ell)}=\left[\begin{array}{lll}
b_{1}^{\ell} & & \\
& \ddots & \\
& & b_{\hat{n}_{\ell}}^{\ell}
\end{array}\right]
$$


and the vector of mass displacements $\mathbf{y}^{(\ell)}(t)=\left[y_{1}^{\ell}(t), \ldots, y_{\hat{n}_{\ell}}^{\ell}(t)\right]^{T}$. The location of the interbuilding TMIDs included in $D^{(\ell)}$ are specified by the list of positions $P^{(\ell)}=\left[p_{1}^{\ell}, \ldots, p_{\hat{n}_{\ell}}^{\ell}\right]$. In this case, the case- and rod-placement matrices $\mathbf{P}_{c}^{(\ell)} \in \mathbb{R}^{n_{1} \times \hat{n}_{\ell}}$ and $\mathbf{P}_{r}^{(\ell)} \in \mathbb{R}^{n_{2} \times \hat{n}_{\ell}}$ can be obtained by extracting the columns indicated in the list of positions $P^{(\ell)}$ from the identity matrices $\mathbf{I}_{n_{1}}$ and $\mathbf{I}_{n_{2}}$, respectively. For the particular interbuilding actuation system $D^{(\ell)}=\left[d_{1}^{\ell}, d_{2}^{\ell}\right]$ in Fig. $1 \mathrm{~b}$, the list of actuator positions is $P^{(\ell)}=[2,4]$, and the corresponding case- and rod-placement matrices have the following form:

$$
\mathbf{P}_{c}^{(\ell)}=\left[\begin{array}{ll}
0 & 0 \\
1 & 0 \\
0 & 0 \\
0 & 1
\end{array}\right], \mathbf{P}_{r}^{(\ell)}=\left[\begin{array}{ll}
0 & 0 \\
1 & 0 \\
0 & 0 \\
0 & 1 \\
0 & 0
\end{array}\right],
$$

which contain the second and fourth columns of $\mathbf{I}_{4}$ and $\mathbf{I}_{5}$, respectively. An overall actuation scheme for a two-building system with $\hat{n}=\hat{n}_{1}+\hat{n}_{2}+\hat{n}_{\ell}$ TMIDs can be written in the form $D=\left[D^{(1)}, D^{(2)}, D^{(\ell)}\right]$. It should be observed that some of the actuation subsystems can be empty. Specifically, the interstory actuation subsystem $D^{(j)}$ will be empty for a non-actuated building $\mathscr{B}^{(j)}$, and an empty interbuilding subsystem $D^{(\ell)}$ will correspond to an unlinked configuration. The overall actuation system $D$ can also be empty in the case of a totally non-actuated two-building system. For an overall linked actuation scheme $D=\left[D^{(1)}, D^{(2)}, D^{(\ell)}\right]$ with $\hat{n}_{\ell}>0$ interbuilding TMIDs, the dynamical response of the actuated two-building system can be described by the second-order model

$$
\tilde{\mathbf{M}} \ddot{\tilde{\mathbf{q}}}(t)+\tilde{\mathbf{C}} \dot{\tilde{\mathbf{q}}}(t)+\tilde{\mathbf{K}} \tilde{\mathbf{q}}(t)=-\tilde{\mathbf{M}}_{w}[\mathbf{1}]_{\tilde{n} \times 1} \ddot{w}_{g}(t),
$$

where $\tilde{\mathbf{q}}(t) \in \mathbb{R}^{\tilde{n} \times 1}$ is the overall extended displacement-vector with the following form:

$$
\tilde{\mathbf{q}}(t)=\left[\begin{array}{c}
\tilde{\mathbf{q}}^{(1)}(t) \\
\tilde{\mathbf{q}}^{(2)}(t) \\
\mathbf{y}^{(\ell)}(t)
\end{array}\right]
$$

and $\tilde{n}=\tilde{n}_{1}+\tilde{n}_{2}+\hat{n}_{\ell}$ is the overall number of degrees-of-freedom corresponding to the actuated two-building system. In this case, the overall generalized mass-matrix $\tilde{\mathbf{M}} \in \mathbb{R}^{\tilde{n} \times \tilde{n}}$ has the following block structure:

$$
\tilde{\mathbf{M}}=\left[\begin{array}{ccc}
\tilde{\mathbf{M}}^{(1)} & {[\mathbf{0}]_{\tilde{n}_{1} \times \tilde{n}_{2}}} & {[\mathbf{0}]_{\tilde{n}_{1} \times \hat{n}_{\ell}}} \\
{[\mathbf{0}]_{\tilde{n}_{2} \times \tilde{n}_{1}}} & \tilde{\mathbf{M}}^{(2)}+\tilde{\mathbf{P}}_{r}^{(\ell)} \mathbf{B}^{(\ell)}\left\{\tilde{\mathbf{P}}_{r}^{(\ell)}\right\}^{T} & -\tilde{\mathbf{P}}_{r}^{(\ell)} \mathbf{B}^{(\ell)} \\
{[\mathbf{0}]_{\hat{n}_{\ell} \times \tilde{n}_{1}}} & -\left\{\tilde{\mathbf{P}}_{r}^{(\ell)} \mathbf{B}^{(\ell)}\right\}^{T} & \hat{\mathbf{M}}^{(\ell)}+\mathbf{B}^{(\ell)}
\end{array}\right],
$$

where $\tilde{\mathbf{M}}^{(1)}$ and $\tilde{\mathbf{M}}^{(2)}$ are the generalized mass matrices of $\mathscr{B}^{(1)}$ and $\mathscr{B}^{(2)}$, respectively, $\hat{\mathbf{M}}^{(\ell)}$ and $\mathbf{B}^{(\ell)}$ are the mass and inertance matrices of the interbuilding actuation system $D^{(\ell)}$, and $\tilde{\mathbf{P}}_{r}^{(\ell)} \in$ 
$\mathbb{R}^{\tilde{n}_{2} \times \hat{n}_{\ell}}$ is the extended rod-placement matrix of $D^{(\ell)}$, which is equal to $\mathbf{P}_{r}^{(\ell)}$ when $\mathscr{B}^{(2)}$ is a nonactuated building and has the form

$$
\tilde{\mathbf{P}}_{r}^{(\ell)}=\left[\begin{array}{c}
\mathbf{P}_{r}^{(\ell)} \\
{[\mathbf{0}]_{\hat{n}_{2} \times \hat{n}_{\ell}}}
\end{array}\right]
$$

when $\mathscr{B}^{(2)}$ is an actuated building equipped with $\hat{n}_{2}>0$ interstory TMIDs. The overall extended damping-matrix $\tilde{\mathbf{C}} \in \mathbb{R}^{\tilde{n} \times \tilde{n}}$ can be written in the form

$$
\tilde{\mathbf{C}}=\left[\begin{array}{ccc}
\tilde{\mathbf{C}}^{(1)}+\tilde{\mathbf{P}}_{c}^{(\ell)} \hat{\mathbf{C}}^{(\ell)}\left\{\tilde{\mathbf{P}}_{c}^{(\ell)}\right\}^{T} & {[\mathbf{0}]_{\tilde{n}_{1} \times \tilde{n}_{2}}} & -\tilde{\mathbf{P}}_{c}^{(\ell)} \hat{\mathbf{C}}^{(\ell)} \\
{[\boldsymbol{0}]_{\tilde{n}_{2} \times \tilde{n}_{1}}} & \tilde{\mathbf{C}}^{(2)} & {[\mathbf{0}]_{\tilde{n}_{2} \times \hat{n}_{\ell}}} \\
-\left\{\tilde{\mathbf{P}}_{c}^{(\ell)} \hat{\mathbf{C}}^{(\ell)}\right\}^{T} & {[\mathbf{0}]_{\hat{n}_{\ell} \times \tilde{n}_{2}}} & \hat{\mathbf{C}}^{(\ell)}
\end{array}\right],
$$

where $\tilde{\mathbf{C}}^{(1)}$ and $\tilde{\mathbf{C}}^{(2)}$ are the respective extended damping matrices of $\mathscr{B}^{(1)}$ and $\mathscr{B}^{(2)} ; \hat{\mathbf{C}}^{(\ell)}$ is the damping matrix of the interbuilding actuation system $D^{(\ell)}$; and $\tilde{\mathbf{P}}_{c}^{(\ell)} \in \mathbb{R}^{\tilde{n}_{1} \times \hat{n}_{\ell}}$ is the extended caseplacement matrix of $D^{(\ell)}$, which is equal to $\mathbf{P}_{c}^{(\ell)}$ when $\mathscr{B}^{(1)}$ is a non-actuated building, and has the form

$$
\tilde{\mathbf{P}}_{c}^{(\ell)}=\left[\begin{array}{c}
\mathbf{P}_{c}^{(\ell)} \\
{[\mathbf{0}]_{\hat{n}_{1} \times \hat{n}_{\ell}}}
\end{array}\right]
$$

when $\mathscr{B}^{(1)}$ is an actuated building equipped with $\hat{n}_{1}>0$ interstory TMIDs. The overall extended stiffness-matrix $\tilde{\mathbf{K}} \in \mathbb{R}^{\tilde{n} \times \tilde{n}}$ has the following block structure:

$$
\tilde{\mathbf{K}}=\left[\begin{array}{ccc}
\tilde{\mathbf{K}}^{(1)}+\tilde{\mathbf{P}}_{c}^{(\ell)} \hat{\mathbf{K}}^{(\ell)}\left\{\tilde{\mathbf{P}}_{c}^{(\ell)}\right\}^{T} & {[\mathbf{0}]_{\tilde{n}_{1} \times \tilde{n}_{2}}} & -\tilde{\mathbf{P}}_{c}^{(\ell)} \hat{\mathbf{K}}^{(\ell)} \\
{[\boldsymbol{0}]_{\tilde{n}_{2} \times \tilde{n}_{1}}} & \tilde{\mathbf{K}}^{(2)} & {[\mathbf{0}]_{\tilde{n}_{2} \times \hat{n}_{\ell}}} \\
-\left\{\tilde{\mathbf{P}}_{c}^{(\ell)} \hat{\mathbf{K}}^{(\ell)}\right\}^{T} & {[\mathbf{0}]_{\hat{n}_{\ell} \times \tilde{n}_{2}}} & \hat{\mathbf{K}}^{(\ell)}
\end{array}\right],
$$

where $\tilde{\mathbf{K}}^{(1)}$ and $\tilde{\mathbf{K}}^{(2)}$ are the extended stiffness matrices of $\mathscr{B}^{(1)}$ and $\mathscr{B}^{(1)}$, respectively, and $\hat{\mathbf{K}}^{(\ell)}$ is the stiffness matrix of $D^{(\ell)}$. Finally, the overall extended mass-matrix $\tilde{\mathbf{M}}_{w} \in \mathbb{R}^{\tilde{n} \times \tilde{n}}$ has the blockdiagonal structure

$$
\tilde{\mathbf{M}}_{w}=\left[\begin{array}{ccc}
\tilde{\mathbf{M}}_{w}^{(1)} & {[\mathbf{0}]_{\tilde{n}_{1} \times \tilde{n}_{2}}} & {[\mathbf{0}]_{\tilde{n}_{1} \times \hat{n}_{\ell}}} \\
{[\mathbf{0}]_{\tilde{n}_{2} \times \tilde{n}_{1}}} & \tilde{\mathbf{M}}_{w}^{(2)} & {[\mathbf{0}]_{\tilde{n}_{2} \times \hat{n}_{\ell}}} \\
{[\mathbf{0}]_{\hat{n}_{\ell} \times \tilde{n}_{1}}} & {[\mathbf{0}]_{\hat{n}_{\ell} \times \tilde{n}_{2}}} & \hat{\mathbf{M}}^{(\ell)}
\end{array}\right] .
$$

For unlinked actuation schemes, the interbuilding actuation system $D^{(\ell)}$ is empty and we have $\hat{n}_{\ell}=0$. In this case, we obtain that the column vector $\mathbf{y}^{(\ell)}(t)$ and the matrices $\hat{\mathbf{M}}^{(\ell)}, \hat{\mathbf{C}}^{(\ell)}, \hat{\mathbf{K}}^{(\ell)}$, $\mathbf{B}^{(\ell)}, \mathbf{P}_{c}^{(\ell)}$ and $\mathbf{P}_{r}^{(\ell)}$ are degenerated empty matrices. By removing these empty matrices from the 
block-matrices in Eqs. (19)-(25), we obtain that the overall extended displacement-vector has the reduced form

$$
\tilde{\mathbf{q}}(t)=\left[\begin{array}{l}
\tilde{\mathbf{q}}^{(1)}(t) \\
\tilde{\mathbf{q}}^{(2)}(t)
\end{array}\right],
$$

and the overall extended matrices have the following reduced block-diagonal structure:

$$
\begin{gathered}
\tilde{\mathbf{M}}=\left[\begin{array}{cc}
\tilde{\mathbf{M}}^{(1)} & {[\mathbf{0}]_{\tilde{n}_{1} \times \tilde{n}_{2}}} \\
{[\mathbf{0}]_{\tilde{n}_{2} \times \tilde{n}_{1}}} & \tilde{\mathbf{M}}^{(2)}
\end{array}\right], \quad \tilde{\mathbf{C}}=\left[\begin{array}{cc}
\tilde{\mathbf{C}}^{(1)} & {[\mathbf{0}]_{\tilde{n}_{1} \times \tilde{n}_{2}}} \\
{[\mathbf{0}]_{\tilde{n}_{2} \times \tilde{n}_{1}}} & \tilde{\mathbf{C}}^{(2)}
\end{array}\right], \\
\tilde{\mathbf{K}}=\left[\begin{array}{cc}
\tilde{\mathbf{K}}^{(1)} & {[\mathbf{0}]_{\tilde{n}_{1} \times \tilde{n}_{2}}} \\
{[\mathbf{0}]_{\tilde{n}_{2} \times \tilde{n}_{1}}} & \tilde{\mathbf{K}}^{(2)}
\end{array}\right], \quad \tilde{\mathbf{M}}_{w}=\left[\begin{array}{cc}
\tilde{\mathbf{M}}_{w}^{(1)} & {[\mathbf{0}]_{\tilde{n}_{1} \times \tilde{n}_{2}}} \\
{[\mathbf{0}]_{\tilde{n}_{2} \times \tilde{n}_{1}}} & \tilde{\mathbf{M}}_{w}^{(2)}
\end{array}\right] .
\end{gathered}
$$

Additionally, the interstory actuation systems $D^{(1)}$ and/or $D^{(2)}$ can also be empty. In this case, the overall displacement vector in Eq. (26) and the diagonal blocks of the overall matrices in Eqs. (27) and (28) can be further reduced as discussed in Section 2.3. Specifically, for a fully non-actuated two-building system, we will have $\hat{n}_{1}=\hat{n}_{2}=\hat{n}_{\ell}=0$ and, after removing the corresponding degenerated empty matrices, the displacement vector $\tilde{\mathbf{q}}(t)$ and the matrices $\tilde{\mathbf{M}}=\tilde{\mathbf{M}}_{w}, \tilde{\mathbf{C}}$ and $\tilde{\mathbf{K}}$ will be reduced to the form given in Eqs. (4) and (5), respectively.

\subsection{Output variables and state-space model}

To evaluate the time response of the actuated two-building system, we will consider two different kinds of output variables: interstory drifts and interbuilding approaches. The vector of interstory drifts $\mathbf{r}_{s}^{(j)}(t)=\left[\left\{r_{s}\right\}_{1}^{j}(t), \ldots,\left\{r_{s}\right\}_{n_{j}}^{j}(t)\right]^{T} \in \mathbb{R}^{n_{j} \times 1}$ contains the relative displacements of consecutive stories in building $\mathscr{B}^{(j)}$, which are defined as follows:

$$
\left\{\begin{array}{l}
\left\{r_{s}\right\}_{1}^{j}(t)=q_{1}^{j}(t), \\
\left\{r_{s}\right\}_{i}^{j}(t)=q_{i}^{j}(t)-q_{i-1}^{j}(t), \quad 1<i \leq n_{j} .
\end{array}\right.
$$

To compute the interstory-drift vector $\mathbf{r}_{s}^{(j)}(t)$, we define an output matrix $\mathbf{C}_{s}^{(j)} \in \mathbb{R}^{n_{j} \times n_{j}}$ with the following band-diagonal structure:

$$
\mathbf{C}_{s}^{(j)}=\left[\begin{array}{rrrrr}
1 & & & & \\
-1 & 1 & & & \\
& -1 & \ddots & & \\
& & \ddots & 1 & \\
& & & -1 & 1
\end{array}\right]
$$


where the elements in the main diagonal are equal to 1, the subdiagonal elements are equal to -1 , and all the other elements are null. Then, the corresponding interstory-drift vector can be computed as

$$
\mathbf{r}_{s}^{(j)}(t)=\tilde{\mathbf{C}}_{s}^{(j)} \tilde{\mathbf{q}}^{(j)}(t),
$$

where $\tilde{\mathbf{q}}^{(j)}(t)$ is the extended displacement-vector of $\mathscr{B}^{(j)}$ given in Eq. (10) and

$$
\left.\tilde{\mathbf{C}}_{s}^{(j)}=\left[\begin{array}{ll}
\mathbf{C}_{s}^{(j)} & {[\mathbf{0}}
\end{array}\right]_{n_{j} \times \hat{n}_{j}}\right]
$$

is the extended interstory-drift output-matrix. The overall interstory-drift vector

$$
\mathbf{r}_{s}(t)=\left[\begin{array}{l}
\mathbf{r}_{s}^{(1)}(t) \\
\mathbf{r}_{s}^{(2)}(t)
\end{array}\right]
$$

can be computed in the form

$$
\mathbf{r}_{s}(t)=\tilde{\mathbf{C}}_{s} \tilde{\mathbf{q}}(t)
$$

with the output matrix

$$
\tilde{\mathbf{C}}_{s}=\left[\begin{array}{ccc}
\tilde{\mathbf{C}}_{s}^{(1)} & {[\mathbf{0}]_{n_{1} \times \tilde{n}_{2}}} & {[\mathbf{0}]_{n_{1} \times \hat{n}_{\ell}}} \\
{[\mathbf{0}]_{n_{2} \times \tilde{n}_{1}}} & \tilde{\mathbf{C}}_{s}^{(2)} & {[\mathbf{0}]_{n_{2} \times \hat{n}_{\ell}}}
\end{array}\right] .
$$

The interbuilding approaching $\left\{r_{a}\right\}_{i}(t)$ describes the approximation between the stories $s_{i}^{1}$ and $s_{i}^{2}$ placed at the same level in the adjacent buildings. The vector of interbuilding approachings $\mathbf{r}_{a}(t) \in \mathbb{R}^{n_{\ell} \times 1}$ contains the elements

$$
\left\{r_{a}\right\}_{i}(t)=-\left(q_{i}^{2}(t)-q_{i}^{1}(t)\right), 1 \leq i \leq n_{\ell},
$$

where $n_{\ell}=\min \left(n_{1}, n_{2}\right)$ is the number of story-levels in the two-building system that can be laterally linked. The interbuilding-approaching vector can be computed in the form

$$
\mathbf{r}_{a}(t)=\tilde{\mathbf{C}}_{a} \tilde{\mathbf{q}}(t)
$$

by using an output-matrix $\tilde{\mathbf{C}}_{a} \in \mathbb{R}^{n_{\ell} \times \tilde{n}}$ with the following structure:

$$
\left.\tilde{\mathbf{C}}_{a}=\left[\begin{array}{lll}
\tilde{\mathbf{C}}_{a}^{(1)} & -\tilde{\mathbf{C}}_{a}^{(2)} & {[\mathbf{0}}
\end{array}\right]_{n_{\ell} \times \hat{n}_{\ell}}\right],
$$

where $\tilde{\mathbf{C}}_{a}^{(j)}$ is a matrix of dimension $n_{\ell} \times \tilde{n}_{j}$ that contains the fist $n_{\ell}$ rows of the identity matrix $\mathbf{I}_{\tilde{n}_{j}}$.

In order to take advantage of the powerful off-the-shelf numerical tools for computing the frequency and time responses, it is convenient to formulate the second-order model in Eq. (18) as a first-order state-space model

$$
\dot{\tilde{\mathbf{x}}}(t)=\tilde{\mathbf{A}} \tilde{\mathbf{x}}(t)+\tilde{\mathbf{B}} \ddot{w}_{g}(t),
$$


with the state vector

$$
\tilde{\mathbf{x}}(t)=\left[\begin{array}{c}
\tilde{\mathbf{q}}(t) \\
\tilde{\mathbf{q}}(t)
\end{array}\right],
$$

and the system matrices

$$
\tilde{\mathbf{A}}=\left[\begin{array}{cc}
{[\mathbf{0}]_{\tilde{n} \times \tilde{n}}} & \mathbf{I}_{\tilde{n}} \\
-\tilde{\mathbf{M}}^{-1} \tilde{\mathbf{K}} & -\tilde{\mathbf{M}}^{-1} \tilde{\mathbf{C}}
\end{array}\right], \quad \tilde{\mathbf{B}}=\left[\begin{array}{c}
{[\mathbf{0}]_{\tilde{n} \times 1}} \\
-\tilde{\mathbf{M}}^{-1} \tilde{\mathbf{M}}_{w}[\mathbf{1}]_{\tilde{n} \times 1}
\end{array}\right] .
$$

In this case, the overall interstory-drift vector $\mathbf{r}_{s}(t)$ can be computed in the form

$$
\mathbf{r}_{S}(t)=\overline{\mathbf{C}}_{s} \tilde{\mathbf{x}}(t),
$$

using the output matrix

$$
\left.\overline{\mathbf{C}}_{s}=\left[\begin{array}{ll}
\tilde{\mathbf{C}}_{s} & {[\mathbf{0}}
\end{array}\right]_{n \times \tilde{n}}\right] .
$$

Analogously, the vector of interbuilding approachings can be obtained in the form

$$
\mathbf{r}_{a}(t)=\overline{\mathbf{C}}_{a} \tilde{\mathbf{x}}(t),
$$

with the output matrix

$$
\left.\overline{\mathbf{C}}_{a}=\left[\begin{array}{ll}
\tilde{\mathbf{C}}_{a} & {[\mathbf{0}}
\end{array}\right]_{n_{\ell} \times \tilde{n}}\right] .
$$

Remark 4. For the fully non-actuated case, a state-space formulation of the second-order model in Eq. (3) can be derived from Eqs. (39)-(41) by removing the degenerate empty matrices. Specifically, we will obtain the state-space model

$$
\dot{\mathbf{x}}(t)=\mathbf{A x}(t)+\mathbf{B} \ddot{w}_{g}(t),
$$

with the state vector

$$
\mathbf{x}(t)=\left[\begin{array}{c}
\mathbf{q}(t) \\
\dot{\mathbf{q}}(t)
\end{array}\right],
$$

and the system matrices

$$
\mathbf{A}=\left[\begin{array}{cc}
{[\mathbf{0}]_{n \times n}} & \mathbf{I}_{n} \\
-\mathbf{M}^{-1} \mathbf{K} & -\mathbf{M}^{-1} \mathbf{C}
\end{array}\right], \quad \mathbf{B}=\left[\begin{array}{c}
{[\mathbf{0}]_{n \times 1}} \\
-[\mathbf{1}]_{n \times 1}
\end{array}\right] .
$$

Remark 5. It should be noted that positive values of the interbuilding approaching $\left\{r_{a}(t)\right\}_{i}$ indicate a reduction of the interbuilding separation at the $i$-th story-level. Consequently, interbuilding collisions can take place for large interbuilding-approaching values. 


\section{Tuning procedure}

\subsection{Parameters optimization}

The characteristics of the interstory actuation system in building $\mathscr{B}^{(j)}$ can be described by the list of actuator positions $P^{(j)}=\left[p_{1}^{j}, \ldots, p_{\hat{n}_{j}}^{j}\right]$ and a list of TMID parameters $\theta^{(j)} \in \mathbb{R}^{1 \times 4 \hat{n}_{j}}$ with the following form:

$$
\boldsymbol{\theta}^{(j)}=\left[\hat{m}_{1}^{j}, \ldots, \hat{m}_{\hat{n}_{j}}^{j}, \hat{c}_{1}^{j}, \ldots, \hat{c}_{\hat{n}_{j}}^{j}, \hat{k}_{1}^{j}, \ldots, \hat{k}_{\hat{n}_{j}}^{j}, b_{1}^{j}, \ldots, b_{\hat{n}_{j}}^{j}\right] .
$$

The interbuilding actuation system can be represented in a similar way by means of the list of positions $P^{(\ell)}=\left[p_{1}^{\ell}, \ldots, p_{\hat{n}_{\ell}}^{\ell}\right]$ and the list of parameters

$$
\theta^{(\ell)}=\left[\hat{m}_{1}^{\ell}, \ldots, \hat{m}_{\hat{n}_{\ell}}^{\ell}, \hat{c}_{1}^{\ell}, \ldots, \hat{c}_{\hat{n}_{\ell}}^{\ell}, \hat{k}_{1}^{\ell}, \ldots, \hat{k}_{\hat{n}_{\ell}}^{\ell}, b_{1}^{\ell}, \ldots, b_{\hat{n}_{\ell}}^{\ell}\right] .
$$

The overall actuation system can be specified by a pair $(\Sigma, \theta)$, where $\Sigma=\left(P^{(1)}, P^{(2)}, P^{(\ell)}\right)$ is a triplet that contains the lists of actuator positions, and $\theta=\left[\theta^{(1)}, \theta^{(2)}, \theta^{(\ell)}\right] \in \mathbb{R}^{1 \times 4 \hat{n}}$ is the overall list of TMID parameters. The triplet of positions lists $\Sigma$ defines the actuation layout, determining the dimensions $\hat{n}_{1}, \hat{n}_{2}$ and $\hat{n}_{\ell}$, the structure of the parameter list $\theta$, and the different case- and rodplacement matrices discussed in the previous section. The list of parameters $\theta$ sets the properties and behavior of the different actuation devices and determines the parameter matrices given in Eqs. (7) and (16) in an obvious way. For a given actuation system $(\Sigma, \theta)$, the dynamical response of the actuated two-building system can be described by the state-space model

$$
\mathscr{S}_{\Sigma, \theta}:\left\{\begin{array}{l}
\dot{\tilde{\mathbf{x}}}(t)=\tilde{\mathbf{A}}_{\Sigma, \theta} \tilde{\mathbf{x}}(t)+\tilde{\mathbf{B}}_{\Sigma, \theta} \ddot{w}_{g}(t), \\
\mathbf{z}(t)=\mathbf{C}_{z} \tilde{\mathbf{x}}(t),
\end{array}\right.
$$

where $\tilde{\mathbf{x}}(t)$ is the extended state-vector in Eq. (40); $\tilde{\mathbf{A}}_{\Sigma, \theta}$ and $\tilde{\mathbf{B}}_{\Sigma, \theta}$ are the extended system-matrices given in Eq. (41); $\mathbf{z}(t)$ is a suitable controlled-output vector, which is used to describe some relevant aspects of the two-building vibrational response; and $\mathbf{C}_{z}$ is the controlled-output matrix. In a single-objective approach, the performance of the actuation system $(\Sigma, \theta)$ is evaluated by means of a real cost-function $J(\Sigma, \theta)$. For a given actuation layout $\Sigma$, the objective of the tuning problem is to determine an optimal parameter list $\theta^{*}$, which can be obtained by solving a constrained optimization problem

$$
\mathscr{P}_{\Sigma, \Theta}:\left\{\begin{array}{l}
\min _{\theta} J(\Sigma, \theta), \\
\text { s.t. } \theta \in \Theta,
\end{array}\right.
$$

where $\Theta$ is a suitable parameter domain. In the seismic protection of closely adjacent buildings, particular attention must be paid to reducing both the interstory drifts and the interbuilding approachings. For buildings with a small number of stories, reduced interstory-drift values always lead to small or moderate interbuilding approachings. Considering this fact, in this work we will focus our attention on reducing the overall interstory-drift response, and will take the vector $\mathbf{r}_{s}(t)$ in Eq. (33) as controlled-output. This vector can be computed from the extended state-vector $\tilde{\mathbf{x}}(t)$ 
using the controlled-output matrix $\overline{\mathbf{C}}_{s}$ given in Eq. (43). To define the cost function $J(\Sigma, \theta)$, we consider the $H_{\infty}$ system-norm of the extended system

$$
J(\Sigma, \theta)=\sup _{0<\left\|\ddot{w}_{g}\right\|_{2}<\infty} \frac{\left\|\mathbf{r}_{s}\right\|_{2}}{\left\|\ddot{w}_{g}\right\|_{2}}
$$

which describes the worst-case energy-gain from the external disturbance $\ddot{w}_{g}(t)$ to the controlledoutput $\mathbf{r}_{s}(t)$. The $H_{\infty}$-norm can be computed in the frequency domain by solving the following optimization problem:

$$
J(\Sigma, \theta)=\sup _{f \in \mathbb{R}}\left\{\sigma_{\max }\left[\mathbf{T}_{\Sigma, \theta}(f)\right]\right\}
$$

where $f$ is the frequency in $\mathrm{Hz} ; \sigma_{\max }[\cdot]$ denotes the maximum singular value; and $\mathbf{T}_{\Sigma, \theta}(f)$ is the frequency-response function (FRF) from the exogenous disturbance to the controlled-output, which can be written in the following form:

$$
\mathbf{T}_{\Sigma, \theta}(f)=\overline{\mathbf{C}}_{s}\left(2 \pi f j \mathbf{I}_{2 \tilde{n}}-\tilde{\mathbf{A}}_{\Sigma, \theta}\right)^{-1} \tilde{\mathbf{B}}_{\Sigma, \theta}
$$

with $j=\sqrt{-1}$. As to the parameter domain $\Theta$, we will set a simple system of constraints of the form $\theta_{L} \leq \theta \leq \theta_{U}$, where $\theta_{L} \in \mathbb{R}^{1 \times 4 \hat{n}}$ and $\theta_{U} \in \mathbb{R}^{1 \times 4 \hat{n}}$ are constant lists of lower and upper bounds, respectively. For simplicity, we will assume that all the actuators of a particular actuation system have similar characteristics and, consequently, the same lower and upper bounds can be selected for each kind of parameter. Thus, for example, the TMID masses can be subjected to constraints of the form:

$$
L_{\hat{m}} \leq \hat{m}_{i}^{j} \leq U_{\hat{m}}, \quad L_{\hat{m}} \leq \hat{m}_{i}^{\ell} \leq U_{\hat{m}}
$$

where $L_{\hat{m}}$ and $U_{\hat{m}}$ are the lower and upper bounds, respectively, for all the TMID mass-coefficients of the actuation system $(\Sigma, \theta)$. Similarly, common pairs of bounds $\left(L_{\hat{c}}, U_{\hat{c}}\right),\left(L_{\hat{k}}, U_{\hat{k}}\right)$ and $\left(L_{b}, U_{b}\right)$ can be used, respectively, for the TMID damping, stiffness and inertance coefficients.

Remark 6. It should be noted that a pure TMD configuration can be obtained by selecting a set of parameter bounds satisfying $L_{\hat{m}}>0$ and $L_{b}=U_{b}=0$. Similarly, a plain TID configuration will be obtained with $L_{\hat{m}}=U_{\hat{m}}=0$ and $L_{b}>0$. Other special cases as undamped or non-elastic configurations are also possible.

\subsection{Numerical results}

To illustrate the computational efficiency of the proposed tuning procedure and the effectiveness of multi-actuator TID vibration control strategies, we consider a four-story building $\mathscr{B}^{(1)}$ adjacent to a five-story building $\mathscr{B}^{(2)}$ with the mass and stiffness characteristics indicated in Table 1 and the following damping matrices (in Ns/m):

$$
\mathbf{C}^{(1)}=10^{5} \times\left[\begin{array}{cccc}
2.6450 & -0.9034 & 0 & 0 \\
-0.9034 & 2.2455 & -0.7915 & 0 \\
0 & -0.7915 & 2.0078 & -0.6715 \\
0 & 0 & -0.6715 & 1.3719
\end{array}\right],
$$




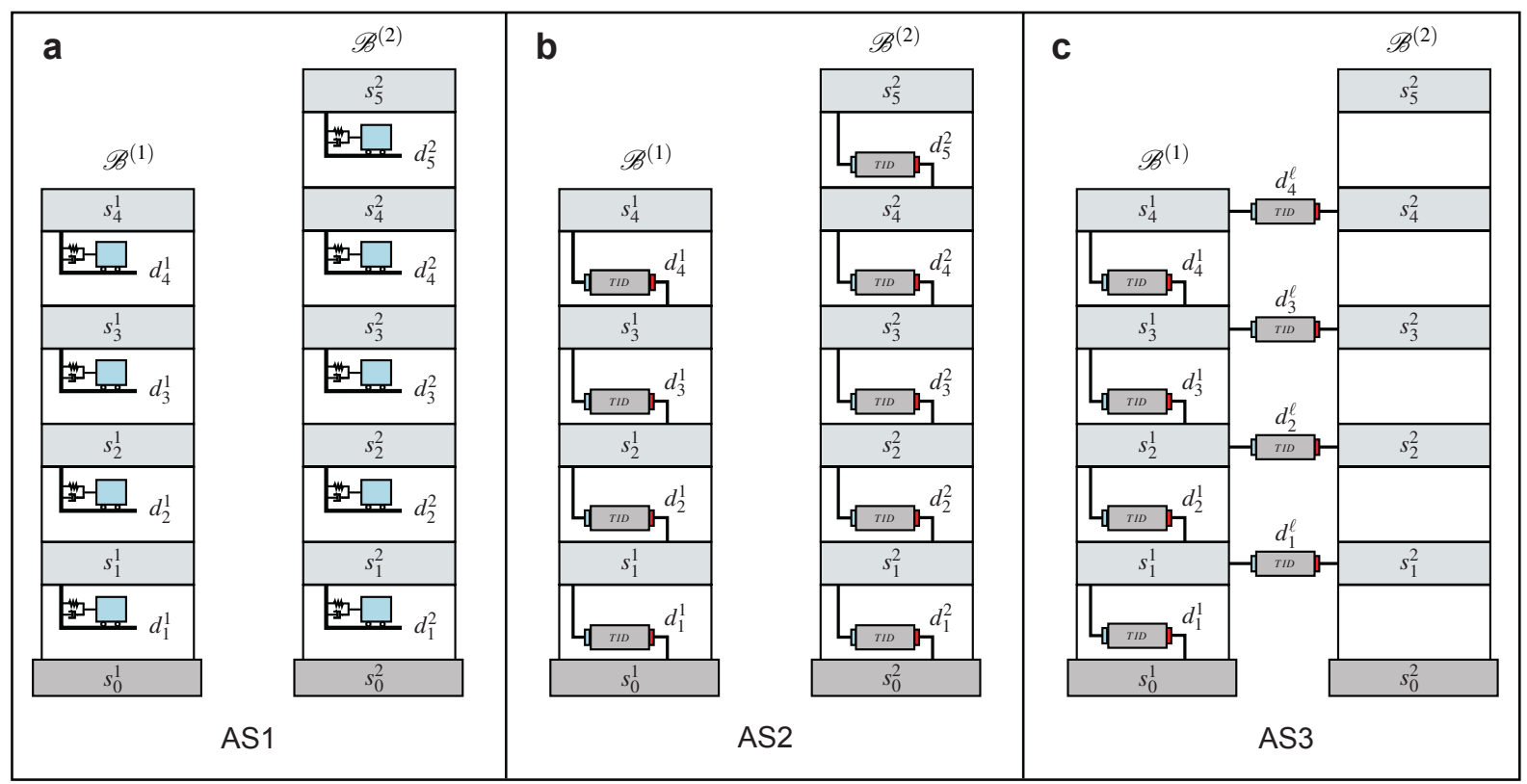

Figure 6: Schematic view of the actuation systems considered in the numerical designs and simulations: (a) Unlinked actuation system AS1 with a full set of interstory TMDs implemented in both buildings. (b) Unlinked actuation system AS2 with a full set of interstory TIDs implemented in both buildings. (c) Linked actuation system AS3 with a full set of interstory TIDs implemented in $\mathscr{B}^{(1)}$, and a complete set of interbuilding TIDs. The building $\mathscr{B}^{(2)}$ is non-actuated in AS3.

$$
\mathbf{C}^{(2)}=10^{5} \times\left[\begin{array}{ccccc}
2.6017 & -0.9244 & 0 & 0 & 0 \\
-0.9244 & 2.1958 & -0.8099 & 0 & 0 \\
0 & -0.8099 & 1.9946 & -0.7281 & 0 \\
0 & 0 & -0.7281 & 1.8670 & -0.6872 \\
0 & 0 & 0 & -0.6872 & 1.2741
\end{array}\right] .
$$

The buildings mass and stiffness coefficients are similar to those presented in [49], and the matrices $\mathbf{C}^{(1)}$ and $\mathbf{C}^{(2)}$ have been computed as Rayleigh damping matrices by setting a $2 \%$ of relative damping on the first and last modes [48]. For these two particular buildings, we consider the actuation systems AS1, AS2 and AS3 schematically displayed in Fig. 6. AS1 is an unlinked actuation system with a full set of interstory TMDs implemented in both buildings. The corresponding actuation layout $\Sigma_{1}$ contains the position lists $P_{1}^{(1)}=[1,2,3,4], P_{1}^{(2)}=[1,2,3,4,5]$ and $P_{1}^{(\ell)}=[]$ (empty list), and the parameter list $\theta_{1}$ has 36 elements. The actuation system AS2 has the same layout as AS1, but, in this case, the actuation devices are TIDs. Finally, AS3 is a linked actuation system with a complete set of interbuilding TIDs and a full set of interstory TIDs implemented in $\mathscr{B}^{(1)}$. In the third case, $\mathscr{B}^{(2)}$ is a non-actuated building, the actuation layout $\Sigma_{3}$ contains the position lists $P_{3}^{(1)}=[1,2,3,4], P_{3}^{(2)}=[]$ and $P_{3}^{(\ell)}=[1,2,3,4]$, and the parameter list $\theta_{3}$ has 32 elements.

To solve the parameter optimization problem $\mathscr{P}_{\Sigma, \Theta}$ in Eq. (52), we have selected the heuristic optimization solver particleswarm ( ) included in the Matlab Global Optimization Toolbox [47], which provides an efficient implementation of the Particle Swarm algorithm with bound constraints. The auxiliary optimization problem in Eq. (54) has been solved with the function 
Table 1: Mass and stiffness coefficients corresponding to the buildings $\mathscr{B}^{(1)}$ and $\mathscr{B}^{(2)}$.

\begin{tabular}{lccccccccc}
\hline story & $s_{1}^{1}$ & $s_{2}^{1}$ & $s_{3}^{1}$ & $s_{4}^{1}$ & $s_{1}^{2}$ & $s_{2}^{2}$ & $s_{3}^{2}$ & $s_{4}^{2}$ & $s_{5}^{2}$ \\
mass $\left(\times 10^{5} \mathrm{Kg}\right)$ & 2.152 & 2.092 & 2.070 & 2.661 & 2.152 & 2.092 & 2.070 & 2.048 & 2.661 \\
stiffness $\left(\times 10^{8} \mathrm{~N} / \mathrm{m}\right)$ & 1.470 & 1.130 & 0.990 & 0.840 & 1.470 & 1.130 & 0.990 & 0.890 & 0.840 \\
\hline
\end{tabular}

Table 2: Values of the optimal parameter configuration $\theta_{1}^{*}$ for the actuation devices of the unlinked TMD actuation system AS1, with an associated optimal cost $J\left(\Sigma_{1}, \theta_{1}^{*}\right)=0.0470$.

\begin{tabular}{lccccccccc}
\hline actuation device & $d_{1}^{1}$ & $d_{2}^{1}$ & $d_{3}^{1}$ & $d_{4}^{1}$ & $d_{1}^{2}$ & $d_{2}^{2}$ & $d_{3}^{2}$ & $d_{4}^{2}$ & $d_{5}^{2}$ \\
mass $\left(\times 10^{4} \mathrm{~kg}\right)$ & 0.019 & 0.050 & 0.040 & 9.798 & 9.999 & 0.012 & 0.010 & 9.996 & 10.000 \\
damping $\left(\times 10^{5} \mathrm{Ns} / \mathrm{m}\right)$ & 1.108 & 9.487 & 1.930 & 2.505 & 0.379 & 9.728 & 9.430 & 1.451 & 2.662 \\
stiffness $\left(\times 10^{7} \mathrm{~N} / \mathrm{m}\right)$ & 2.634 & 1.068 & 0.098 & 0.327 & 0.122 & 0.163 & 2.662 & 0.160 & 0.260 \\
inertance $\left(\times 10^{5} \mathrm{~kg}\right)$ & 0 & 0 & 0 & 0 & 0 & 0 & 0 & 0 & 0 \\
\hline
\end{tabular}

hinfnorm( ) included in the Matlab Robust Control Toolbox [50], which facilitates a fast computation of the $H_{\infty}$ system norm. To compute an optimal parameter list $\theta_{1}^{*}$ for the TMD actuation system AS1, we have considered the optimization problem $\mathscr{P}_{\Sigma_{1}, \Theta_{1}}$ with the parameter domain $\Theta_{1}$ defined by the following bounds:

$$
L_{\hat{m}}=10^{2}, U_{\hat{m}}=10^{5} ; L_{\hat{c}}=10^{4}, U_{\hat{c}}=10^{6} ; L_{\hat{k}}=5 \times 10^{5}, U_{\hat{k}}=5 \times 10^{7} ; L_{b}=U_{b}=0,
$$

where the mass and inertance bounds are in $\mathrm{kg}$, the damping bounds are in Ns/m, and the stiffness bounds are in $\mathrm{N} / \mathrm{m}$. As mentioned in Remark 6, the inertance bounds $L_{b}=U_{b}=0$ enforce a plain TMD configuration. The obtained solution $\theta_{1}^{*}$ attains an optimal $\cos t J\left(\Sigma_{1}, \theta_{1}^{*}\right)=0.0470$ and has the particular parameter values collected in Table 2. For the actuation systems AS2 and AS3 we have selected the parameter domains $\Theta_{2}$ and $\Theta_{3}$ determined by the following set of parameter bounds:

$$
L_{\hat{m}}=U_{\hat{m}}=0 ; \quad L_{\hat{c}}=10^{4}, U_{\hat{c}}=10^{6} ; L_{\hat{k}}=5 \times 10^{5}, U_{\hat{k}}=5 \times 10^{7} ; L_{b}=10^{4}, U_{b}=10^{6},
$$

which maintains the values of the damping and stiffness bounds of $\Theta_{1}$, enforces a pure TID configuration by setting null mass-bounds, and settles a maximum inertance value of $10^{6} \mathrm{~kg}$ with an allowed variation range of two orders of magnitude. For the unlinked TID actuation system AS2, solving the optimization problem $\mathscr{P}_{\Sigma_{2}, \Theta_{2}}$ produces an optimal cost $J\left(\Sigma_{2}, \theta_{2}^{*}\right)=0.0419$ and a list of parameters $\theta_{2}^{*}$ with the values presented in Table 3 . Finally, for the linked TID actuation system AS3, we obtain the optimal cost $J\left(\Sigma_{3}, \theta_{3}^{*}\right)=0.0490$ and a list of parameters $\theta_{3}^{*}$ with the values indicated in Table 4 by solving the optimization problem $\mathscr{P}_{\Sigma_{3}, \Theta_{3}}$.

To provide an intuitive view of the behavior exhibited by the designed actuation systems, a proper set of frequency-response plots have been displayed in Fig. 7. Specifically, the curves in Fig. 7 present the maximum singular-values corresponding to the FRFs $\mathbf{T}_{\Sigma_{1}, \theta_{1}^{*}}(f)$ (blue dotted line), $\mathbf{T}_{\Sigma_{2}, \theta_{2}^{*}}(f)$ (red dash-dotted line) and $\mathbf{T}_{\Sigma_{3}, \theta_{3}^{*}}(f)$ (thick green solid line). As a reference, the plot corresponding to the FRF of the non-actuated two-building system has also been included using a thin black solid line, and is named Free in the legend. The value of the largest peak in these 
Table 3: Values of the optimal parameter configuration $\theta_{2}^{*}$ for the actuation devices of the unlinked TID actuation system AS2, with an associated optimal cost $J\left(\Sigma_{2}, \theta_{2}^{*}\right)=0.0419$.

\begin{tabular}{lccccccccc}
\hline actuation device & $d_{1}^{1}$ & $d_{2}^{1}$ & $d_{3}^{1}$ & $d_{4}^{1}$ & $d_{1}^{2}$ & $d_{2}^{2}$ & $d_{3}^{2}$ & $d_{4}^{2}$ & $d_{5}^{2}$ \\
mass $\left(\times 10^{4} \mathrm{~kg}\right)$ & 0 & 0 & 0 & 0 & 0 & 0 & 0 & 0 & 0 \\
damping $\left(\times 10^{5} \mathrm{Ns} / \mathrm{m}\right)$ & 9.982 & 7.633 & 8.711 & 9.976 & 8.330 & 9.962 & 9.999 & 8.930 & 10.000 \\
stiffness $\left(\times 10^{7} \mathrm{~N} / \mathrm{m}\right)$ & 3.747 & 1.907 & 2.629 & 2.716 & 2.856 & 2.057 & 2.231 & 1.968 & 0.050 \\
inertance $\left(\times 10^{5} \mathrm{~kg}\right)$ & 6.325 & 9.954 & 8.444 & 6.209 & 4.480 & 6.066 & 8.903 & 10.000 & 8.023 \\
\hline
\end{tabular}

Table 4: Values of the optimal parameter configuration $\theta_{3}^{*}$ for the actuation devices of the linked TID actuation system AS3, with an associated optimal cost $J\left(\Sigma_{3}, \theta_{3}^{*}\right)=0.0490$.

\begin{tabular}{lcccccccc}
\hline actuation device & $d_{1}^{1}$ & $d_{2}^{1}$ & $d_{3}^{1}$ & $d_{4}^{1}$ & $d_{1}^{\ell}$ & $d_{2}^{\ell}$ & $d_{3}^{\ell}$ & $d_{4}^{\ell}$ \\
mass $\left(\times 10^{4} \mathrm{~kg}\right)$ & 0 & 0 & 0 & 0 & 0 & 0 & 0 & 0 \\
damping $\left(\times 10^{5} \mathrm{Ns} / \mathrm{m}\right)$ & 9.966 & 9.232 & 9.973 & 9.287 & 8.827 & 0.717 & 2.349 & 9.956 \\
stiffness $\left(\times 10^{7} \mathrm{~N} / \mathrm{m}\right)$ & 3.015 & 2.507 & 2.950 & 2.923 & 0.258 & 1.024 & 5.000 & 0.708 \\
inertance $\left(\times 10^{5} \mathrm{~kg}\right)$ & 9.617 & 9.995 & 9.395 & 9.861 & 9.082 & 4.315 & 2.436 & 3.302 \\
\hline
\end{tabular}

frequency-response plots is the corresponding $H_{\infty}$ system norm, which has the value 0.3611 for the non-actuated system, and is equal to the associated optimal cost for the actuated configurations. Looking at the plots in the figure, two main facts can be clearly appreciated: (i) a remarkable reduction in the $H_{\infty}$-norm (superior to $86.4 \%$ ) is produced by the three designed actuation systems with respect to the non-actuated configuration, and (ii) a significantly better performance is attained by the TID actuation systems AS2 and AS3 in the secondary resonant peaks with respect to the TMD actuation system AS1. The former of these facts confirms the efficiency of the proposed tuning procedure, and illustrates the enhanced performance that can be achieved by multi-actuator control strategies; the later indicates the superior ability of TIDs in mitigating the vibrational response to broad-band excitations.

Remark 7. The selection of proper parameter bounds is a relevant element in the tuning procedure. The particular values presented in Eqs. (59) and (60) have been selected as follows: For the TMD masses, we have set a maximum value of $U_{\hat{m}}=10^{5} \mathrm{Kg}$, which is about $50 \%$ of the story masses. This large mass upper-bound has been intendedly selected to obtain an ideal TMD multi-actuator system with high-performance characteristics [51], which can be taken as a reference in the performance assessment of the proposed TID multi-actuator systems. For the TMD damping and stiffness coefficients, we have selected the upper bounds $U_{\hat{c}}=10^{6} \mathrm{Ns} / \mathrm{m}$ and $U_{\hat{k}}=5.0 \times 10^{7} \mathrm{~N} / \mathrm{m}$, respectively. These values have been adjusted by observing the frequencyresponse characteristics of the obtained TMD multi-actuation system. To facilitate a meaningful comparison, the same stiffness and damping upper-bounds have been used in the TID designs. For the TID inertances, we have assumed that the inerter devices can provide a mass amplificationfactor of two or more orders of magnitude [2]. Accordingly, we have selected the inertance upperbound $U_{b}=10^{6} \mathrm{Kg}$, which implies that the actual mass of the required inerter elements would be, 

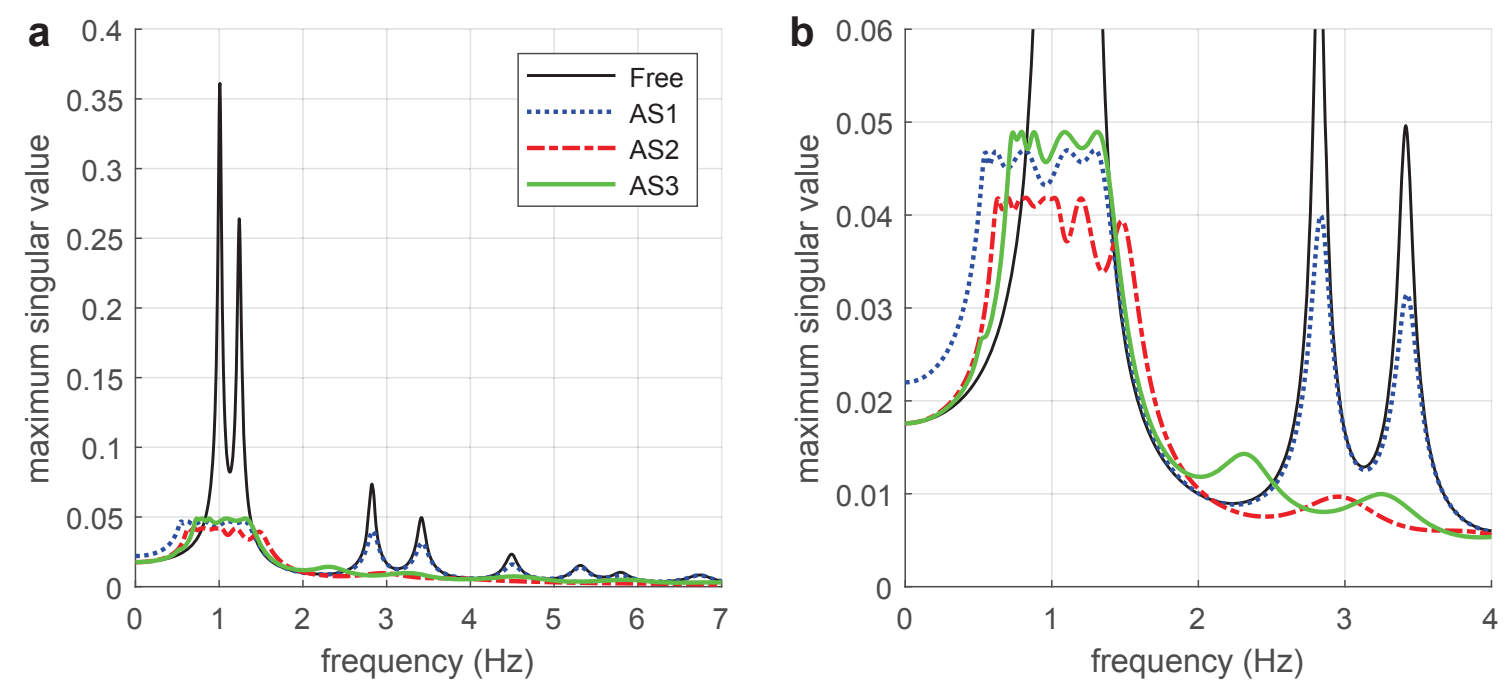

Figure 7: Interstory-drift frequency-response plots corresponding to the non-actuated configuration (thin black solid line), the unlinked TMD actuation system AS1 with parameter list $\theta_{1}^{*}$ (blue dotted line), the unlinked TID actuation system AS2 with parameter list $\theta_{2}^{*}$ (red dash-dotted line), and the linked TID actuation system AS3 with parameter list $\theta_{3}^{*}$ (thick green solid line): (a) Overall view. (b) Close view of the main and secondary resonant peaks.

in the worst case, around a 5\% of the story masses. This choice allows designing TID actuation systems that are a light-weight alternative to the massive high-performance TMDs. After setting the upper-bound values, the lower bounds $L_{\hat{c}}=10^{4} \mathrm{Ns} / \mathrm{m}, L_{\hat{k}}=5.0 \times 10^{5} \mathrm{~N} / \mathrm{m}$ and $L_{b}=10^{4} \mathrm{~kg}$ have been computed by allowing a variation range of two orders of magnitude, which have proved to be large enough to produce positive results and sufficiently narrow for computational efficiency. The TMD masses have required a broader variation range, and the corresponding lower bound has been set to $L_{\hat{m}}=10^{2} \mathrm{Kg}$.

Remark 8. Due to the random characteristics of the Particle Swarm optimization algorithm, the optimal parameter values corresponding to an actuation system $(\Sigma, \theta)$ have been obtained by running a batch of 25 independent instances of the associated optimization problem $\mathscr{P}_{\Sigma, \Theta}$, which produces a set of parameter lists $\left\{\theta^{*}\right\}_{i}$ with associated cost values $J\left(\Sigma,\left\{\theta^{*}\right\}_{i}\right)$. The optimal parameter list $\theta^{*}$ is then obtained by setting $J\left(\Sigma, \theta^{*}\right)=\min _{i} J\left(\Sigma,\left\{\theta^{*}\right\}_{i}\right)$.

Remark 9. The values SwarmSize=75, TolFun=1e-4 and MaxIterations $=200$ have been used for the Particle Swarm solver. Also, the value tol=1e-4 has been set for the relative accuracy of the hinfnorm( ) function. With these options, the computation time required to solve a single instance of the constrained optimization problem $\mathscr{P}_{\Sigma, \Theta}$ has usually varied in the range 90-150 s. Computing an optimal parameter list $\theta^{*}$ by solving a batch of 25 independent instances of $\mathscr{P}_{\Sigma, \Theta}$ has required an overall computation time of about $50 \mathrm{~min}$. The computations discussed in this paper have been carried out using a regular laptop with an Intel ${ }^{\odot}$ Core $^{T M}$ i7-2640M processor at $2.80 \mathrm{GHz}$. 

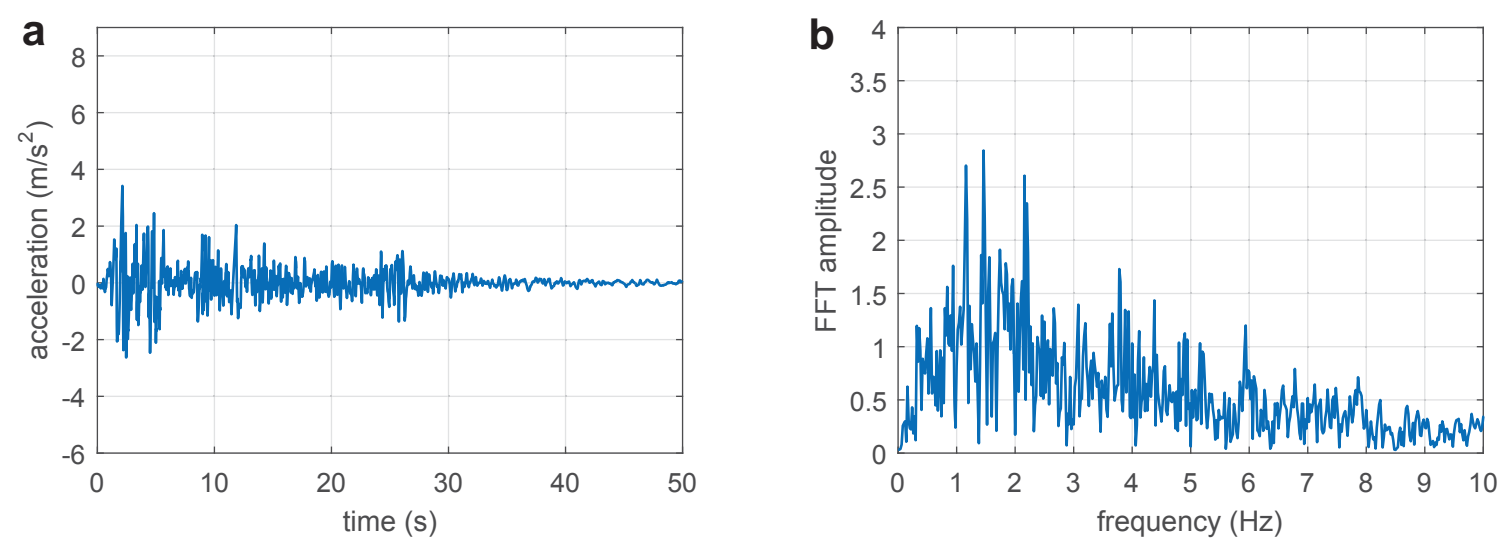

Figure 8: Full-scale North-South El Centro 1940 seismic record with a ground acceleration peak of $3.4170 \mathrm{~m} / \mathrm{s}^{2}$. Recorded at the El Centro substation during the Imperial Valley earthquake, California, 18th May, 1940. (a) Ground acceleration time history. (b) Acceleration spectrum: Fast Fourier Transform amplitude.

Table 5: Percentages of reduction in the maximum interstory-drift and interbuilding-approaching peak-values (with respect to the maximum peak-values of the non-actuated two-building system) obtained for the full-scale North-South El Centro 1940 seismic record.

\begin{tabular}{lccc}
\hline actuation scheme & unlinked TMD AS1 & unlinked TID AS2 & linked TID AS3 \\
interstory drifts building 1 & 38.27 & 43.16 & 44.20 \\
interstory drifts building 2 & 55.52 & 58.10 & 45.89 \\
interbuilding approachings & 71.34 & 75.48 & 80.86 \\
\hline
\end{tabular}

\section{Seismic response}

In this section, we perform a proper set of numerical simulations to demonstrate some relevant features of the seismic responses produced by the designed TMD and TID multi-actuator systems. To this end, we consider two ground acceleration records with different seismic characteristics [52, 53]: (i) the full-scale North-South El Centro 1940 seismic record, which is a medium-size seismic disturbance with a ground acceleration peak of $3.4170 \mathrm{~m} / \mathrm{s}^{2}$ (see Fig. 8); and (ii) the fullscale North-South Northridge 1994 seismic record (see Fig. 10), which is a strong near-fault seismic excitation with a ground acceleration peak of $8.2676 \mathrm{~m} / \mathrm{s}^{2}$. For these two seismic disturbances and the particular two-building system previously considered, we have computed the vectors of interstory drifts $\mathbf{r}_{s}^{(1)}(t)$ and $\mathbf{r}_{s}^{(2)}(t)$, and the vector of interbuilding approachings $\mathbf{r}_{a}(t)$ corresponding to the non-actuated configuration and the actuation systems AS1, AS2 and AS3 designed in Section 3.2. The seismic response of the non-actuated configuration has been obtained using the state-space model in Eqs. (46)-(48), and the response of the actuated configurations has been computed using the corresponding extended state-space models with the form given in Eqs. (39)-(41). The output matrices $\overline{\mathbf{C}}_{s}$ and $\overline{\mathbf{C}}_{a}$ in Eqs. (43) and (45) have been respectively used to obtain the interstory-drift and interbuilding-approaching vectors. To summarize the information provided by the output vectors $\mathbf{r}_{s}^{(1)}(t), \mathbf{r}_{s}^{(2)}(t)$ and $\mathbf{r}_{a}(t)$, we have computed the absolute interstory-drift peak- 
(a) Building 1

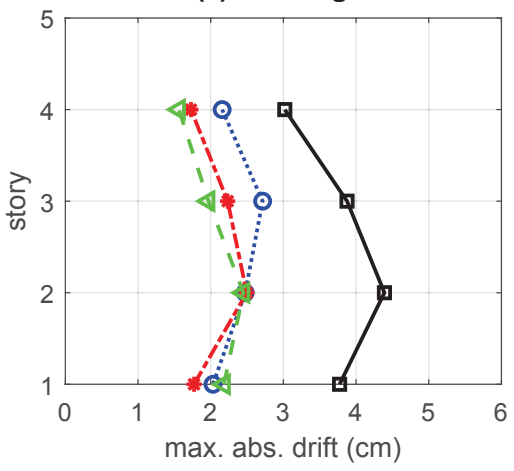

(b) Interbuilding

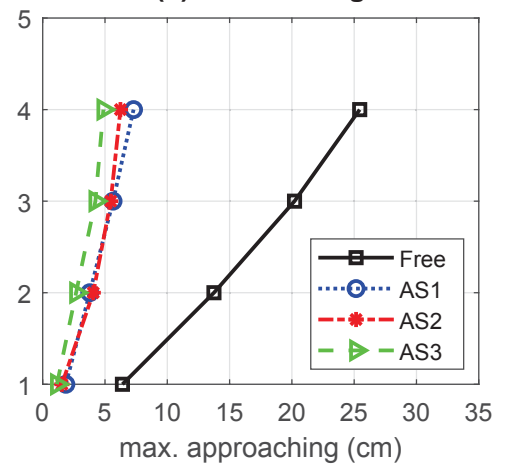

(c) Building 2

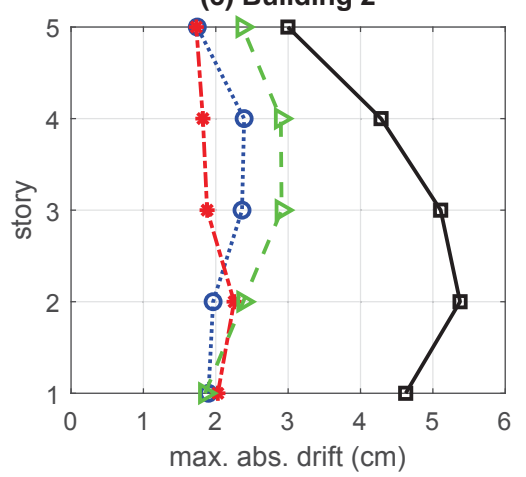

Figure 9: Time responses corresponding to the full-scale North-South El Centro 1940 seismic record. Interstory-drift and interbuilding-approaching peak-values obtained for the non-actuated configuration (black solid line with squares), the unlinked TMD actuation system AS1 (blue dotted line with circles), the unlinked TID actuation system AS2 (red dash-dotted line with asterisks), and the linked TID actuation system AS3 (green dashed line with triangles).

values

$$
\left\{r_{s}^{*}\right\}_{i}^{1}=\max _{t \geq 0}\left|\left\{r_{s}\right\}_{i}^{1}(t)\right|, i=1, \ldots, n_{1}, \quad\left\{r_{s}^{*}\right\}_{i}^{2}=\max _{t \geq 0}\left|\left\{r_{s}\right\}_{i}^{2}(t)\right|, i=1, \ldots, n_{2},
$$

and the interbuilding-approaching peak-values

$$
\left\{r_{a}^{*}\right\}_{i}=\max _{t \geq 0}\left(\left\{r_{a}\right\}_{i}(t)\right), i=1, \ldots, n_{\ell} .
$$

Finally, we have also obtained the maximum interstory-drift and interbuilding-approaching peakvalues

$$
\left\{r_{s}^{*}\right\}^{1}=\max _{1 \leq i \leq n_{1}}\left\{r_{s}^{*}\right\}_{i}^{1}, \quad\left\{r_{s}^{*}\right\}^{2}=\max _{1 \leq i \leq n_{2}}\left\{r_{s}^{*}\right\}_{i}^{2}, \quad r_{a}^{*}=\max _{1 \leq i \leq n_{\ell}}\left\{r_{a}^{*}\right\}_{i}
$$

For the El Centro seismic excitation, the peak-values of the absolute interstory-drifts obtained in building $\mathscr{B}^{(1)}$ and building $\mathscr{B}^{(2)}$ are respectively presented in Figs. 9a and 9c, and the corresponding interbuilding-approaching peak-values are displayed in the central Fig. 9b. In these plots, the black solid lines with squares represent the non-actuated configuration (named Free in the legend), the blue dotted lines with circles pertain to the unlinked TMD actuation system AS1, the red dash-dotted lines with asterisks correspond to the unlinked TID actuation system AS2, and the green dashed lines with triangles show the response of the linked TID actuation system AS3. The plots in Fig. 9 evidence that the three actuation systems attain a good level of reduction in both the interstory-drift and interbuilding-approaching peak-values with respect to the free (non-actuated) response. The percentages of reduction in the maximum interstory-drift and interbuilding-approaching peak-values attained by the considered actuation systems with respect to the maximum peak-values of the free response are collected in Table 5. Thus, for instance, the maximum interstory-drift peak-value of the free response in building $\mathscr{B}^{(1)}$ is produced at the second story-level and has the value $\left\{r_{s}^{*}\right\}_{\text {Free }}^{1}=4.39 \mathrm{~cm}$ (see Fig. 9a). For the TMD actuation 

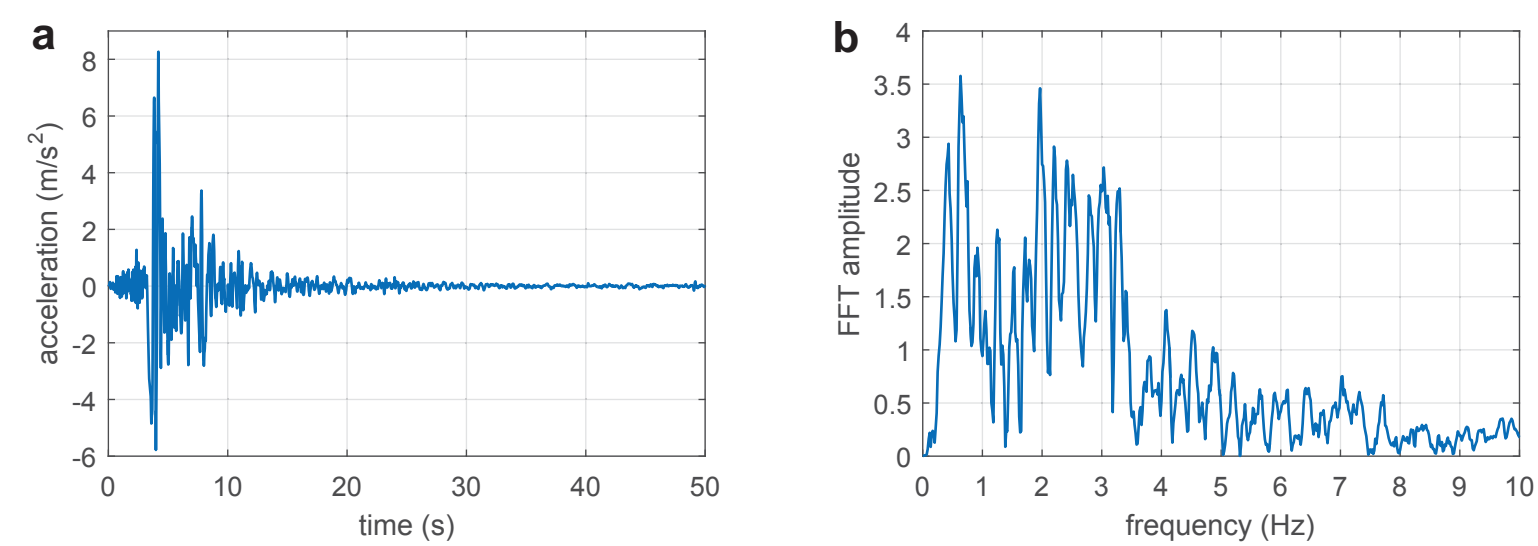

Figure 10: Full-scale North-South Northridge 1994 seismic record with a ground acceleration peak of $8.2676 \mathrm{~m} / \mathrm{s}^{2}$. Recorded at the parking lot of the Sylmar County Hospital, Sylmar, California, during the Northridge earthquake, 17th January, 1994. (a) Ground acceleration time history. (b) Acceleration spectrum: Fast Fourier Transform amplitude.

(a) Building 1

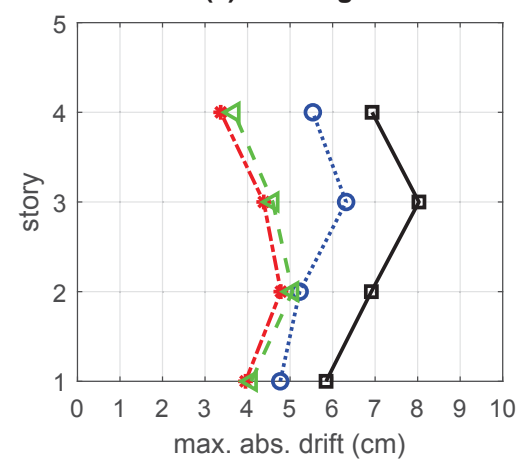

(b) Interbuilding

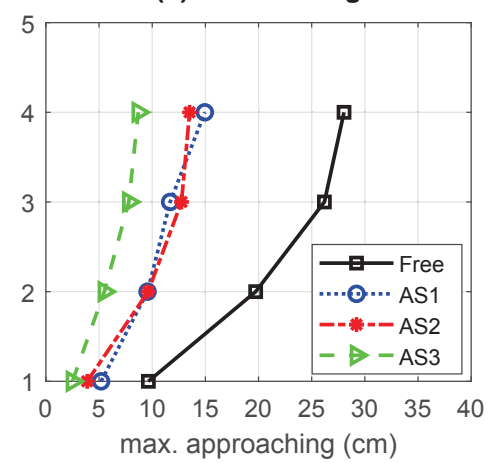

(c) Building 2

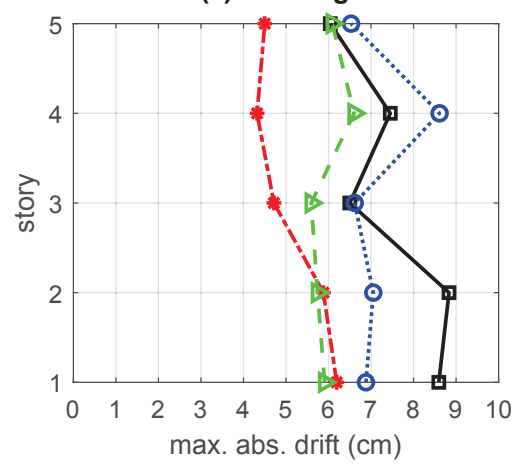

Figure 11: Time responses corresponding to the full-scale North-South Northridge 1994 seismic record. Interstorydrift and interbuilding-approaching peak-values obtained for the non-actuated configuration (black solid line with squares), the unlinked TMD actuation system AS1 (blue dotted line with circles), the unlinked TID actuation system AS2 (red dash-dotted line with asterisks), and the linked TID actuation system AS3 (green dashed line with triangles).

system AS1, the maximum interstory-drift peak-value in building $\mathscr{B}^{(1)}$ is observed at the third story-level and has the value of $\left\{r_{s}^{*}\right\}_{\text {AS1 }}^{1}=2.71 \mathrm{~cm}$, which represents a reduction of $38.27 \%$ with respect to the maximum peak-value of the free-response. From the values in Table 5, it can be appreciated that the unlinked TID actuation system AS2 attains the largest percentage of reduction in the maximum interstory-drift peak-value of building $\mathscr{B}^{(2)}(58.10 \%)$. The linked TID actuation system AS3 achieves the largest reductions in the maximum interstory-drift peak-value of building $\mathscr{B}^{(1)}(44.20 \%)$ and the maximum interbuilding-approaching peak-value $(80.86 \%)$. The unlinked TMD actuation system AS1 also produces significant percentages of reduction in the maximum peak-values, specially for the interstory-drifts of building $\mathscr{B}^{(2)}(55.52 \%)$ and the interbuilding approachings $(71.34 \%)$. However, it should be noted that, despite the large TMD mass-values, the results obtained by the TMD actuation system are uniformly inferior to those produced by the associated TID actuation system AS2. 
Table 6: Percentages of reduction in the maximum interstory-drift and interbuilding-approaching peak-values (with respect to the maximum peak-values of the non-actuated two-building system) obtained for the full-scale North-South Northridge 1994 seismic record.

\begin{tabular}{lccc}
\hline actuation scheme & unlinked TMD AS1 & unlinked TID AS2 & linked TID AS3 \\
interstory drifts building 1 & 21.32 & 40.39 & 37.01 \\
interstory drifts building 2 & 2.61 & 29.90 & 25.26 \\
interbuilding approachings & 46.70 & 51.81 & 69.03 \\
\hline
\end{tabular}

For the Northridge seismic excitation, the obtained plots of interstory-drifts and interbuildingapproaching peak-values are displayed in Fig. 11 using the same colors, line styles and symbols. The corresponding percentages of reduction in the maximum interstory-drift and interbuildingapproaching peak-values with respect to the free response are collected in Table 6. In this case, the plots in Fig. 11 show a partial loss of performance of the TID actuation systems AS2 and AS3 and a more severe degradation in the response of the TMD actuation system AS1, specially in building $\mathscr{B}^{(2)}$. The values in Table 6 indicate that the percentage of reduction in the maximum interstorydrift peak-value attained by the TMD actuation system AS1 in building $\mathscr{B}^{(2)}$ for this near-fault seismic disturbance is only of $2.61 \%$. For the same building, the percentages of reduction produced by the TID actuation systems AS2 and AS3 are $29.90 \%$ and $25.26 \%$, respectively. In fact, for this second type of seismic disturbance, the results obtained by the TID actuation systems are all noticeably superior to those produced by the ideal TMD actuation system.

Remark 10. The seismic responses presented in this section have been computed assuming that the interbuilding gap is large enough to avoid pounding. This approach allows avoiding the modeling and computational difficulties associated to interbuilding impacts. In this case, the maximum interbuilding-approaching peak-value $r_{a}^{*}$ can be understood as a lower bound of safe interbuilding separation. Thus, for example, the maximum interbuilding-approaching peak-values (in $\mathrm{cm}$ ) produced by the actuation scheme AS3 and the non-actuated configurations for the Northridge seismic disturbance are $\left\{r_{a}^{*}\right\}_{\mathrm{AS} 3}=8.69$ and $\left\{r_{a}^{*}\right\}_{\text {Free }}=28.04$, respectively (see Fig. 11b). This means that, for the considered seismic disturbance, an interbuilding gap of $9 \mathrm{~cm}$ can be considered a safe interbuilding separation for the actuated system AS3, while an interbuilding gap of $27 \mathrm{~cm}$ would have produced interbuilding collisions in the non-actuated configuration.

\section{Conclusions and future directions}

In this work, we have studied the problem of designing systems of distributed Tuned MassInerter dampers (TMIDs) for the seismic protection of adjacent multi-story buildings. In order to deal with this complex problem, we have first formulated a mathematical model that allows describing the overall dynamical response of two-building multi-story systems equipped with a distributed set of interstory and/or interbuilding TMID actuators. Next, we have provided a computational tuning procedure that permits obtaining instances of this kind of distributed TMID actuation systems with high-performance characteristics. To illustrate the flexibility and effectiveness of the proposed approach, we have considered a particular two-building system equipped with three different multi-actuator schemes: (i) an unlinked actuation scheme AS1, with a full set of interstory 
Tuned Mass Dampers (TMDs) implemented in both buildings; (ii) an unlinked actuation scheme AS2, with a full set of interstory Tuned Inerter Dampers (TIDs) implemented in both buildings; and (iii) a linked actuation scheme AS3, with a full set of interstory TIDs implemented in only one of the buildings and a complete set of interbuilding TIDs. Configurations AS1 and AS2 facilitate comparing the behavior of TIDs and classical TMDs. Configuration AS3 illustrates the effectiveness of linked actuation schemes in avoiding interbuilding pounding, and the potential of partially actuated schemes. The obtained numerical results indicate the superior performance and robustness of the inerter-based actuation systems. Specifically, the frequency-response plots clearly show the effectiveness of the TID actuation schemes AS2 and AS3 in mitigating the vibrational response in the secondary resonant peaks. Also, the time-response data demonstrate the improved resistance of the inerter-based actuation systems against near-fault seismic disturbances. After the positive results obtained in the present work, the problem of devising an effective methodology for the optimal allocation of interstory and/or interbuilding TID actuation devices in sparsely distributed multi-actuation systems appears as a natural research extension. This problem includes two critical requirements: (i) defining a suitable criterion of optimality, and (ii) determining a viable computational procedure. In this context, using a constrained multi-objective optimization approach seems to be a promising line of solution. However, due to the huge computational cost, the currently available optimization tools cannot be directly applied to designs with a large number of stories and, consequently, more sophisticated and effective procedures need to be developed in order to address this challenging problem.

\section{Acknowledgements}

This work was partially supported by the Spanish Ministry of Economy and Competitiveness under Grant DPI2015-64170-R (MINECO/FEDER).

\section{References}

[1] M. C. Smith, Synthesis of mechanical networks: The inerter, IEEE T. Automat. Contr. 47 (2002) 1648-1662.

[2] C. Papageorgiou, N. E. Houghton, M. C. Smith, Experimental Testing and Analysis of Inerter Devices, J. Dyn. Sys. Meas. Control 131 (2009) 1-11.

[3] M. Z. Q. Chen, C. Papageorgiou, F. Scheibe, F.-C. Wang, M. C. Smith, The missing mechanical circuit element, IEEE Circuits Syst. Mag. 9 (2009) 10-26.

[4] S. J. Swift, M. C. Smith, A. R. Glover, C. Papageorgiou, B. Gartner, N. E. Houghton, Design and modelling of a fluid inerter, Int. J. Control 86 (2013) 2035-2051.

[5] X. Liu, J. Z. Jiang, B. Titurus, A. J. Harrison, D. McBryde, Testing and modelling of the damping effects for fluid-based inerters, Procedia Eng. 199 (2017) 435-440.

[6] X. Liu, J. Z. Jiang, B. Titurus, A. Harrison, Model identification methodology for fluid-based inerters, Mech. Syst. Sig. Process. 106 (2018) 479-494.

[7] Z. Ge, W. Wang, Modeling, testing, and characteristic analysis of a planetary flywheel inerter, Shock Vib. Article ID 2631539 (2018) 1-12.

[8] T. Xu, M. Liang, C. Li, S. Yang, Design and analysis of a shock absorber with variable moment of inertia for passive vehicle suspensions, J. Sound Vib. 355 (2015) 66-85.

[9] M. Lazarek, P. Brzeski, P. Perlikowski, Design and identification of parameters of tuned mass damper with inerter which enables changes of inertance, Mech. Mach. Theory 119 (2018) 161-173.

[10] Y. Hu, M. Z. Q. Chen, S. Xu, Y. Liu, Semiactive inerter and its application in adaptive tuned vibration absorbers, IEEE T. Contr. Syst. T. 25 (2017) 294-300. 
[11] M. Z. Q. Chen, Y. Hu, C. Li, G. Chen, Application of semi-active inerter in semi-active suspensions via force tracking, J. Vib. Acoust. 138 (2016) 1-11.

[12] Y. Shen, L. Chen, Y. Liu, X. Zhang, Modeling and optimization of vehicle suspension employing a nonlinear fluid inerter, Shock Vib. Article ID 2623017 (2016) 1-9.

[13] Y. Hu, M. Z. Q. Chen, Y. Sun, Comfort-oriented vehicle suspension design with skyhook inerter configuration, J. Sound Vib. 405 (2017) 34-47.

[14] X.-L. Zhang, T. Zhang, J. Nie, L. Chen, A semiactive skyhook-inertance control strategy based on continuously adjustable inerter, Shock Vib. Article ID 6828621 (2018) 1-8.

[15] A. Kras, P. Gardonio, Velocity feedback control with a flywheel proof mass actuator, J. Sound Vib. 402 (2017) 31-50.

[16] A. Siami, A. Cigada, H. R. Karimi, E. Zappa, Vibration protection of a famous statue against ambient and earthquake excitation using a tuned inerter-damper, Machines 5 (2017) 1-19.

[17] J. Guo, H. Wen, Y. Li, K. Zhang, Y. Liu, Design and performance analysis of anti-sway \& anti-vibration system for marine operating table with inerter, IOP Conf. Ser.: Earth Environ. Sci. 128 (2018) 1-5.

[18] A. Siami, H. R. Karimi, A. Cigada, E. Zappa, E. Sabbioni, Parameter optimization of an inerter-based isolator for passive vibration control of Michelangelo's Rondanini Pietà, Mech. Syst. Sig. Process. 98 (2018) 667-683.

[19] N. Alujević, D. Čakmak, H. Wolf, M. Jokić, Passive and active vibration isolation systems using inerter, J. Sound Vib. 418 (2018) 163-183.

[20] Y. Li, J. Z. Jiang, S. A. Neild, H. Wang, Optimal inerter-based shock-strut configurations for landing-gear touchdown performance, J. Aircr. 54 (2017) 1901-1909.

[21] R. Takano, M. Yamakita, Q. Zhu, Analysis of biped running with rotational inerter, in: Proceedings of the 2016 IEEE International Conference on Robotics and Biomimetics, Qingdao, China, 2016, pp. 1424-1429.

[22] M. Z. Q. Chen, K. Shen, C. Zhai, A biomechanical model of human muscular-skeletal system with inertial effects, in: Proceedings of the 35th Chinese Control Conference, Chengdu, China, 2016, pp. 9331-9336.

[23] X. Fang, K.-C. Chuang, X. Jin, Z. Huang, Band-gap properties of elastic metamaterials with inerter-based dynamic vibration absorbers, J. Appl. Mech. 85 (2018) 1-9.

[24] M. Z. Q. Chen, K. Wang, Y. Zou, J. Lam, Realization of a special class of admittances with one damper and one inerter for mechanical control, IEEE T. Automat. Contr. 58 (2013) 1841-1846.

[25] I. F. Lazar, S. A. Neild, D. J. Wagg, Using an inerter-based device for structural vibration suppression, Earthquake Eng. Struct. Dyn. 43 (2014) 1129-1147.

[26] L. Marian, A. Giaralis, Optimal design of a novel tuned mass-damper-inerter (TMDI) passive vibration control configuration for stochastically support-excited structural systems, Probab. Eng. Mech. 38 (2014) 156-164.

[27] A. Giaralis, F. Petrini, Optimum design of the tuned mass-damper-inerter for serviceability limit state performance in wind-excited tall buildings, Procedia Eng. 199 (2017) 1773-1778.

[28] R. Ruiz, A. Giaralis, A. Taflanidis, D. Lopez-Garcia, Risk-informed optimization of the tuned mass-damperinerter (TMDI) for seismic protection of buildings in Chile, in: Proceedings of the 16th World Conference on Earthquake Engineering, Santiago, Chile, 2017, pp. 1-12.

[29] D. De Domenico, G. Ricciardi, An enhanced base isolation system equipped with optimal tuned mass damper inerter (TMDI), Earthquake Eng. Struct. Dyn. 47 (2018) 1169-1192.

[30] D. De Domenico, N. Impollonia, G. Ricciardi, Soil-dependent optimum design of a new passive vibration control system combining seismic base isolation with tuned inerter damper, Soil Dyn. Earthquake Eng. 105 (2018) 37-53.

[31] D. De Domenico, G. Ricciardi, Earthquake protection of existing structures with limited seismic joint: base isolation with supplemental damping versus rotational inertia, Adv. Civ. Eng. Article ID 6019495 (2018) 1-24.

[32] A. Giaralis, A. A. Taflanidis, Optimal tuned mass-damper-inerter (TMDI) design for seismically excited MDOF structures with model uncertainties based on reliability criteria, Struct. Control Hlth. Monit. 25 (2018) 1-22.

[33] X. Shi, S. Zhu, Dynamic characteristics of stay cables with inerter dampers, J. Sound Vib. 423 (2018) $287-305$.

[34] V. H. Cu, B. Han, D. H. Pham, W. T. Yan, Free vibration and damping of a taut cable with an attached viscous mass damper, KSCE J. Civ. Eng. 22 (2018) 1792-1802.

[35] S. Zhang, R. Zhang, Z. Zhao, Demand-based optimal design of storage tank with inerter system, Shock Vib. Article ID 2956153 (2017) 1-14. 
[36] Y. Hu, M. Z. Q. Chen, Passive structural control with inerters for a floating offshore wind turbine, in: Proceedings of the 36th Chinese Control Conference, Dalian, China, 2017, pp. 9266-9271.

[37] Y. Hu, J. Wang, M. Z. Q. Chen, Z. Li, Y. Sun, Load mitigation for a barge-type floating offshore wind turbine via inerter-based passive structural control, Eng. Struct. 177 (2018) 198-209.

[38] J. Salvi, A. Giaralis, Concept study of a novel energy harvesting-enabled tuned mass-damper-inerter (EH-TMDI) device for vibration control of harmonically-excited structures, J. Phys. Conf. Ser. 744 (2016) 1-12.

[39] T. Asai, Y. Watanabe, Outrigger tuned inertial mass electromagnetic transducers for high-rise buildings subject to long period earthquakes, Eng. Struct. 153 (2017) 404-410.

[40] Y. Luo, H. Sun, X. Wang, L. Zuo, N. Chen, Wind induced vibration control and energy harvesting of electromagnetic resonant shunt tuned mass-damper-inerter for building structures, Shock Vib. Article ID 4180134 (2017) 1-13.

[41] T. Asai, Y. Araki, K. Ikago, Structural control with tuned inertial mass electromagnetic transducers, Struct. Control Hlth. Monit. 25 (2018) 1-18.

[42] F. Palacios-Quiñonero, J. Rubió-Massegú, J. M. Rossell, H. R. Karimi, Semiactive-passive structural vibration control strategy for adjacent structures under seismic excitation, J. Franklin Inst. 349 (2012) 3003-3026.

[43] F. Palacios-Quiñonero, J. Rubió-Massegú, J. M. Rossell, H. R. Karimi, Integrated design of hybrid interstoryinterbuilding multi-actuation schemes for vibration control of adjacent buildings under seismic excitations, App. Sci. 7 (2017) 1-23.

[44] S. Y. Zhang, T. D. Lewis, J. Z. Jiang, S. A. Neild, Passive vibration suppression using multiple inerter-based devices for a multi-storey building structure, in: Proceedings of the 6th European Conference on Structural Control (EACS 2016), Sheffield, England, Paper No. 146, 2016, pp. 1-11.

[45] A. Y. Leung, H. Zhang, C. C. Cheng, Y. Y. Lee, Particle swarm optimization of TMD by non-stationary base excitation during earthquake, Earthquake Eng. Struct. Dyn. 37 (2008) 1223-1246.

[46] A. Y. Leung, H. Zhang, Particle swarm optimization of tuned mass dampers, Eng. Struct. 31 (2009) $715-728$.

[47] Global Optimization Toolbox R2017b, MathWorks Inc., Natick, MA, 2017.

[48] A. K. Chopra, Dynamics of Structures. Theory and Applications to Earthquake Engineering, 3rd ed., Prentice Hall, Upper Saddle River, NJ, USA, 2007.

[49] N. Kurata, T. Kobori, M. Takahashi, N. Niwa, H. Midorikawa, Actual seismic response controlled building with semi-active damper system, Earthquake Eng. Struct. Dyn. 28 (1999) 1427-1447.

[50] G. Balas, R. Chiang, A. Packard, M. Safonov, Robust Control Toolbox R2017b, MathWorks Inc., Natick, MA, 2017.

[51] P. Xiang, A. Nishitani, Seismic vibration control of building structures with multiple tuned mass damper floors integrated, Earthquake Eng. Struct. Dyn. 43 (2014) 909-925.

[52] B. F. Spencer Jr., R. E. Christenson, S. J. Dyke, Next generation benchmark control problem for seismically excited buildings, in: Proceedings of the 2nd World Conference on Structural Control, Kyoto, Japan, 1999, pp. $1-41$.

[53] Y. Ohtori, R. E. Christenson, B. F. Spencer Jr., S. J. Dyke, Benchmark control problems for seismically excited nonlinear buildings, J. Eng. Mech. 130 (2004) 366-385. 